\title{
Novel Investigation of Fractional-Order Cauchy-Reaction Diffusion Equation Involving Caputo-Fabrizio Operator
}

\author{
Meshari Alesemi, ${ }^{1}$ Naveed Iqbal $\mathbb{D}^{2},{ }^{2}$ and Mohammed S. Abdo $\mathbb{D}^{3}$ \\ ${ }^{1}$ Department of Mathematics, College of Science, University of Bisha, P. O. Box 511, Bisha 61922, Saudi Arabia \\ ${ }^{2}$ Department of Mathematics, Faculty of Science, University of Ha'il, Ha'il 2440, Saudi Arabia \\ ${ }^{3}$ Department of Mathematics, Hodeidah University, Al-Hodeidah, Yemen
}

Correspondence should be addressed to Naveed Iqbal; n.iqbal@uoh.edu.sa and Mohammed S. Abdo; msabdo@hoduniv.net.ye

Received 23 November 2021; Revised 14 December 2021; Accepted 18 December 2021; Published 27 January 2022

Academic Editor: Mahmut I ik

Copyright (c) 2022 Meshari Alesemi et al. This is an open access article distributed under the Creative Commons Attribution License, which permits unrestricted use, distribution, and reproduction in any medium, provided the original work is properly cited.

\begin{abstract}
In this article, the new iterative transform technique and homotopy perturbation transform method are applied to calculate the fractional-order Cauchy-reaction diffusion equation solution. Yang transformation is mixed with the new iteration method and homotopy perturbation method in these methods. The fractional derivative is considered in the sense of Caputo-Fabrizio operator. The convection-diffusion models arise in physical phenomena in which energy, particles, or other physical properties are transferred within a physical process via two processes: diffusion and convection. Four problems are evaluated to demonstrate, show, and verify the present methods' efficiency. The analytically obtained results by the present method suggest that the method is accurate and simple to implement.
\end{abstract}

\section{Introduction}

The convection-diffusion equation is a mixture of convection and diffusion equations and identifies physical processes where energy, particles, or other physical properties are transmitted inside a physical process due to two process steps: diffusion and convection. In standard form, the convection-diffusion model is written as follows:

$$
\frac{\partial \mathbb{U}}{\partial \mathfrak{I}}=\nabla \cdot(D \cdot \nabla \mathbb{U})-\nabla \cdot(\vec{v} \mathbb{U})+R
$$

where $D$ is the diffusivity, $\mathbb{U}$ is the variable term, such as thermal diffusivity for heat flow or mass diffusion coefficient for particle, and $\vec{v}$ is the average velocity that the volume is travelling. For instance, in convection, $u$ might be the density of river in salt and then the flow velocity of water $\vec{v}$. For example, in a calm lake, $\vec{v}$ would be the average velocity of bubbles rising to the surface due to buoyancy, and $\mathbb{U}$ would be the concentration of small bubbles. $R$ defines "sinks" or "sources" of the quantity $\mathbb{U}$. For a chemical species, $R>0$ indicates that a chemical reaction is increasing the number of the species, while $R>0$ indicates that a chemical reaction is decreasing the number of the species. If thermal energy is generated by friction, $R>0$ may occur in heat transport. $\nabla$ denotes gradient, while $\nabla$. denotes divergence. Previously, different techniques have been applied to investigate these models such as Adomian's decomposition technique [1], variational iteration technique [2], Bessel collocation technique [3], and homotopy perturbation technique [4].

In recent decades, fractional derivatives have been used to interpret many physical problems mathematically, and these representations have produced excellent results in modelling real-world issues. Many basic definitions of fractional operators were given by Riesz, Riemann-Liouville, Hadamard, Weyl, Grunwald-Letnikov, Liouville-Caputo, Caputo-Fabrizio, and Atangana-Baleanu, among others [5-8]. Over the last few years, many nonlinear equations have been developed and widely used in nonlinear physical sciences like chemistry, biology, mathematics, and different 
branches of physics like plasma physics, condensed matter physics, fluid mechanics, field theory, and nonlinear optics. The exact outcome of nonlinear equations is crucial in determining the characteristics and behaviour of physical processes. Still, it is impossible to find exact results when dealing with linear equations. Many useful methods have been applied to investigate nonlinear fractional partial differential equations, for example, analytical solutions with the help of natural decomposition method of fractional-order heat and wave equations [9], fractional-order partial differential equations with proportional delay [10], fractional-order hyperbolic telegraph equation [11] and fractional-order diffusion equations [12], the variational iterative transform method [13], the homotopy perturbation transform method [14, 15], the homotopy analysis transform method [16, 17], reduced differential transform method $[18,19]$, qhomotopy analysis transform method [20-24], the finite element technique [25], the finite difference technique [26], and so on [27-30].

Daftardar-Gejji and Jafari developed a new iterative method of analysis for solving nonlinear equations in $2006[31,32]$. It is the first application of Laplace transformation in iterative technique by Jafari et al. Iterative Laplace transformation method [33] was introduced as a simple method for estimating approximate effects of the fractional partial differential equation system. Iterative Laplace transformation method (NITM) is used to solve linear and nonlinear partial differential equations such as fractional-order Fornberg Whitham equations [34], time-fractional Zakharov Kuznetsov equation [35], and fractional-order Fokker Planck equations [36].

In 1999, He developed the homotopy perturbation method (HPM) [37], which combines the homotopy technique, and the standard perturbation method has been broadly utilized to both linear and nonlinear models [38-40]. The homotopy perturbation method is important because it eliminates the need for a small parameter in the model, eliminating the disadvantages of traditional perturbation techniques. The main goal of this paper is to use HPM to solve nonlinear fractional-order Cauchy-reaction diffusion equation using a newly introduced integral transformation known as the "Yang transform" [41]. The suggested technique is applied to analyse two well-known nonlinear partial differential equations. In the context of a quickly convergent series, we obtain a power series solution, and only a few iterations are required to obtain very efficient solutions. There is no need for a discretization technique or linearization for the nonlinear equations, and just a few few can yield a result that can be quickly estimated to utilize these methods.

\section{Basic Definitions}

We provide the fundamental definitions that will be used throughout the article. For the purpose of simplification, we write the exponential decay kernel as, $K(\mathfrak{I}, \mathrm{\varrho})=$ $e^{[-\wp(\mathfrak{\Im}-\varrho / 1-\wp)]}$.
Definition 1. The Caputo-Fabrizio derivative is given as follows [42]:

$$
{ }^{\mathrm{CF}} D_{\Im}^{\wp}[\mathbb{P}(\mathfrak{\Im})]=\frac{N(\wp)}{1-\wp} \int_{0}^{\mathfrak{\Im}} \mathbb{P}^{\prime}(\mathrm{\varrho}) K(\mathfrak{I}, \mathrm{\varrho}) d \mathrm{\varrho}, n-1<\wp \leq n .
$$

$N(\wp)$ is the normalization function with $N(0)=N(1)=1$.

$$
{ }^{\mathrm{CF}} D_{\mathfrak{J}}^{\mathfrak{J}}[\mathbb{P}(\mathfrak{I})]=\frac{N(\wp)}{1-\wp} \int_{0}^{\mathfrak{I}}[\mathbb{P}(\mathfrak{I})-\mathbb{P}(\mathrm{\varrho})] K(\mathfrak{I}, \mathrm{\varrho}) d \mathrm{\varrho} .
$$

Definition 2. The fractional integral Caputo-Fabrizio is given as [42]

$$
{ }^{\mathrm{CF}} I_{\mathfrak{J}}^{\mathfrak{S}}[\mathbb{P}(\mathfrak{I})]=\frac{1-\wp}{N(\wp)} \mathbb{P}(\mathfrak{I})+\frac{\wp}{N(\wp)} \int_{0}^{\mathfrak{J}} \mathbb{P}(\varrho) d \varrho, \mathfrak{J} \geq 0, \wp \in(0,1] .
$$

Definition 3. For $N(\wp)=1$, the following result shows the Caputo-Fabrizio derivative of Laplace transformation [42]:

$$
L\left[{ }^{\mathrm{CF}} D_{\mathfrak{\Im}}^{\mathfrak{S}}[\mathbb{P}(\mathfrak{I})]\right]=\frac{v L[\mathbb{P}(\mathfrak{I})-\mathbb{P}(0)]}{v+\wp(1-v)} .
$$

Definition 4. The Yang transformation of $\mathbb{P}(\mathfrak{\Im})$ is expressed as [42]

$$
\mathbb{Y}[\mathbb{P}(\mathfrak{I})]=\chi(v)=\int_{0}^{\infty} \mathbb{P}(\mathfrak{I}) e^{-\frac{\mathfrak{T}}{v}} d \mathfrak{I} . \mathfrak{I}>0,
$$

Remark 5. Yang transformation of few useful functions is defined as below.

$$
\begin{aligned}
\mathbb{Y}[1] & =v, \\
\mathbb{Y}[\mathfrak{I}] & =v^{2}, \\
\mathbb{Y}\left[\mathfrak{J}^{i}\right] & =\Gamma(i+1) v^{i+1} .
\end{aligned}
$$

Lemma 6 (Laplace-Yang duality). Let the Laplace transformation of $\mathbb{P}(\mathfrak{I})$ be $F(v)$, and then, $\chi(v)=F(1 / v)$ [43].

Proof. From Equation (5), we can achieve another type of the Yang transformation by putting $\mathfrak{\Im} / v=\zeta$ as

$$
L[\mathbb{P}(\Im)]=\chi(v)=v \int_{0}^{\infty} \mathbb{P}(v \zeta) e^{\zeta} d \zeta . \zeta>0 .
$$

Since $L[\mathbb{P}(\mathfrak{J})]=F(v)$, this implies that

$$
F(v)=L[\mathbb{P}(\mathfrak{I})]=\int_{0}^{\infty} \mathbb{P}(\mathfrak{I}) e^{-v \Im} d \mathfrak{I} .
$$

Put $\mathfrak{J}=\zeta / v$ in (8), and we have

$$
F(v)=\frac{1}{v} \int_{0}^{\infty} \mathbb{P}\left(\frac{\zeta}{v}\right) e^{\zeta} d \zeta
$$


Thus, from Equation (7), we achieve

$$
F(v)=\chi\left(\frac{1}{v}\right)
$$

Also from Equations (5) and (8), we achieve

$$
F\left(\frac{1}{v}\right)=\chi(v)
$$

The connections (10) and (11) represent the duality link between the Laplace and Yang transformation.

Lemma 7. Let $\mathbb{P}(\mathfrak{I})$ be a continuous function; then, the Caputo-Fabrizio derivative Yang transformation of $\mathbb{P}(\mathfrak{I})$ is define by [43]

$$
\mathbb{Y}[\mathbb{P}(\mathfrak{I})]=\frac{\mathbb{Y}[\mathbb{P}(\mathfrak{I})-v \mathbb{P}(0)]}{1+\wp(v-1)}
$$

Proof. The Caputo-Fabrizio fractional Laplace transformation is given by

$$
L[\mathbb{P}(\mathfrak{I})]=\frac{L[v \mathbb{P}(\mathfrak{J})-\mathbb{P}(0)]}{v+\wp(1-v)} .
$$

Also, we have that the connection among Laplace and Yang property, i.e., $\chi(v)=F(1 / v)$. To achieve the necessary result, we substitute $v$ by $1 / v$ in Equation (13), and we get

$$
\begin{aligned}
& \mathbb{P}(\mathfrak{I})]=\frac{1 / v \mathbb{Y}[\mathbb{P}(\mathfrak{I})-\mathbb{P}(0)]}{1 / v+\wp(1-1 / v)}, \\
& \mathbb{P}[\mathbb{I})]=\frac{\mathbb{Y}[\mathbb{P}(\mathfrak{I})-v \mathbb{P}(0)]}{1+\wp(v-1)} .
\end{aligned}
$$

The proof is completed.

\section{Algorithm of the HPTM}

The procedure of general nonlinear Caputo-Fabrizio fractional partial differential equations is through HPTM. Let us take a general nonlinear Caputo-Fabrizio partial differential equations with nonlinear function $N(\mathbb{U}(\varphi, \mathfrak{J}))$ and linear fractional $L(\mathbb{U}(\varphi, \mathfrak{\Im}))$ as $[43]$

$$
\left\{\begin{array}{l}
\operatorname{CF}_{\mathfrak{J}}^{\rho} \mathbb{V}(\varphi, \mathfrak{I})+L(\mathbb{V}(\varphi, \mathfrak{I}))+N(\mathbb{V}(\varphi, \mathfrak{J}))=g(\varphi, \mathfrak{J}), \\
\mathbb{V}(\varphi, 0)=h(\varphi)
\end{array}\right.
$$

where the term $g(\varphi, \mathfrak{I})$ shows the source function. Using Yang transformation to Equation (16), one can obtain

$$
\begin{aligned}
& \frac{\mathbb{Y}[\mathbb{V}(\varphi, \mathfrak{\Im})-v \mathbb{V}(\varphi, 0)]}{1+\wp(v-1)} \\
& =-\mathbb{Y}[L(\mathbb{V}(\varphi, \mathfrak{\Im}))+N(\mathbb{V}(\varphi, \mathfrak{I}))]+\mathbb{Y}[g(\varphi, \mathfrak{\Im})]
\end{aligned}
$$

$$
\begin{aligned}
\mathbb{Y}[\mathbb{V}(\varphi, \mathfrak{J})]= & v h(\varphi)-(1+\wp(v-1)) \\
& \cdot[\mathbb{Y}[L(\mathbb{V}(\varphi, \mathfrak{I}))+N(\mathbb{V}(\varphi, \mathfrak{J}))]+\mathbb{Y}[g(\varphi, \mathfrak{I})]
\end{aligned}
$$

Implementing inverse Yang transformation, we obtain

$$
\begin{aligned}
\mathbb{V}(\varphi, \mathfrak{I})= & \mathbb{V}(\varphi, 0)-\mathbb{Y}^{-1}[1+\wp(\nu-1) \\
& \cdot[\mathbb{Y}[L(\mathbb{V}(\varphi, \mathfrak{J}))+N(\mathbb{V}(\varphi, \mathfrak{J}))]+\mathbb{Y}[g(\varphi, \mathfrak{J})]],
\end{aligned}
$$

where the term $\mathbb{V}(\varphi, \mathfrak{I})$ shows the source function and with the initial condition. Now, we apply homoptopy perturbation method.

$$
\mathbb{V}(\varphi, \mathfrak{J})=\sum_{i=0}^{\infty} \rho^{i} \mathbb{V}_{i}(\varphi, \mathfrak{I})
$$

We decompose the nonlinear term $N(\mathbb{V}(\varphi, \mathfrak{\Im}))$ as

$$
N(\mathbb{V}(\varphi, \mathfrak{\Im}))=\sum_{i=0}^{\infty} \rho^{i} H_{i}(\mathbb{V})
$$

where $H_{i}(\mathbb{V})$ represents the He's polynomial and is calculated through the following formula:

$H_{i}\left(\mathbb{V}_{1}, \mathbb{V}_{2}, \mathbb{V}_{3}, \cdots, \mathbb{V}_{i}\right)=\frac{1}{\Gamma(i+1)} \frac{\partial^{i}}{\partial \rho^{i}}\left[N\left(\sum_{i=0}^{\infty} \rho^{i} \mathbb{V}_{i}\right)\right]_{\rho=0}, i=1,2,3$.

Substituting Equations (19) and (20) in Equation (18), we obtain

$$
\begin{aligned}
\sum_{i=0}^{\infty} \rho^{i} \mathbb{V}_{i}(\varphi, \mathfrak{I})= & \mathbb{V}(\varphi, \mathfrak{I})-\rho\left(\mathbb{V}^{-1}[(1+\wp(v-1)) \mathbb{Y}\right. \\
\cdot & {\left.\left.\left[L \sum_{i=0}^{\infty} \rho_{i}^{i \mathbb{V}}(\varphi, \mathfrak{I})+N \sum_{i=0}^{\infty} \rho^{i} H_{i}(\mathbb{V})\right]\right]\right) }
\end{aligned}
$$

We obtain the following terms by coefficients comparing of $\rho$ in (22):

$$
\begin{aligned}
\rho^{0}: \mathbb{V}_{0}(\varphi, \mathfrak{I}) & =\mathbb{V}(\varphi, \mathfrak{I}), \\
\rho^{1}: \mathbb{V}_{1}(\varphi, \mathfrak{I}) & =\mathbb{V}^{-1}\left[(1+\wp(v-1)) \mathbb{Y}\left[L\left(\mathbb{V}_{0}(\varphi, \mathfrak{I})\right)+H_{0}(\mathbb{V})\right]\right] \\
\rho^{2}: \mathbb{V}_{2}(\varphi, \mathfrak{I}) & =\mathbb{V}^{-1}\left[(1+\wp(v-1)) \mathbb{Y}\left[L\left(\mathbb{V}_{1}(\varphi, \mathfrak{I})\right)+H_{1}(\mathbb{V})\right]\right] \\
\rho^{3}: \mathbb{V}_{3}(\varphi, \mathfrak{I}) & =\mathbb{V}^{-1}\left[(1+\wp(v-1)) \mathbb{Y}\left[L\left(\mathbb{V}_{2}(\varphi, \mathfrak{I})\right)+H_{2}(\mathbb{V})\right]\right] \\
& \vdots \\
\rho^{i}: \mathbb{V}_{i}(\varphi, \mathfrak{I}) & =\mathbb{V}^{-1}\left[(1+\wp(v-1)) \mathbb{Y}\left[L\left(\mathbb{V}_{i}(\varphi, \mathfrak{I})\right)+H_{i}(\mathbb{V})\right]\right]
\end{aligned}
$$


As a result, the obtained solution of Equation (16) can be written as follows:

$$
\mathbb{V}(\varphi, \mathfrak{J})=\mathbb{V}_{0}(\varphi, \mathfrak{J})+\mathbb{V}_{1}(\varphi, \mathfrak{J})+\cdots
$$

\section{Error Analysis and Convergence}

The following theorems are fundamental on the techniques address the original models [16] error analysis and convergence.

Theorem 8. Let $\mathbb{V}(\varphi, \mathfrak{\Im})$ be the actual result of $(16)$, and let $\mathbb{V}_{i}(\varphi, \mathfrak{J}) \in H$ and $\sigma \in(0,1)$, where $H$ denotes the Hilbert space. Then, the achieved result $\sum_{i=0}^{\infty} \mathbb{V}{ }_{i}(\varphi, \mathfrak{I})$ will converge $\mathbb{V}(\varphi, \mathfrak{J})$ if $\mathbb{V}_{i}(\varphi, \mathfrak{I}) \leq \mathbb{V}_{i-1}(\varphi, \mathfrak{J}) \forall i>A$, i.e., for any $\omega>0 \exists A$ $>0$, such that $\left\|\mathbb{V}_{i+n}(\varphi, \mathfrak{I})\right\| \leq \beta, \forall i, n \in N[43]$.

Proof. We make a sequence of $\sum_{i=0}^{\infty} \mathbb{V}_{i}(\varphi, \mathfrak{\Im})$.

$C_{0}(\varphi, \mathfrak{I})=\mathbb{V}_{0}(\varphi, \mathfrak{I})$,

$C_{1}(\varphi, \mathfrak{I})=\mathbb{V}_{0}(\varphi, \mathfrak{I})+\mathbb{V}_{1}(\varphi, \mathfrak{I})$,

$C_{2}(\varphi, \mathfrak{J})=\mathbb{V}_{0}(\varphi, \mathfrak{J})+\mathbb{V}_{1}(\varphi, \mathfrak{J})+\mathbb{V}_{2}(\varphi, \mathfrak{J})$,

$C_{3}(\varphi, \mathfrak{I})=\mathbb{V}_{0}(\varphi, \mathfrak{I})+\mathbb{V}_{1}(\varphi, \mathfrak{I})+\mathbb{V}_{2}(\varphi, \mathfrak{I})+\mathbb{V}_{3}(\varphi, \mathfrak{I})$

$C_{i}(\varphi, \mathfrak{J})=\mathbb{V}_{0}(\varphi, \mathfrak{J})+\mathbb{V}_{1}(\varphi, \mathfrak{J})+\mathbb{V}_{2}(\varphi, \mathfrak{J})+\cdots+\mathbb{V}_{i}(\varphi, \mathfrak{J})$

To provide the correct outcome, we have to demonstrate that $C_{i}(\varphi, \mathfrak{\Im})$ forms a "Cauchy sequence." Take, for example,

$$
\begin{aligned}
\left\|C_{i+1}(\varphi, \mathfrak{I})-C_{i}(\varphi, \mathfrak{I})\right\| & =\left\|\mathbb{V}_{i+1}(\varphi, \mathfrak{I})\right\| \leq \sigma\left\|\mathbb{V}_{i}(\varphi, \mathfrak{I})\right\| \\
& \leq \sigma^{2}\left\|\mathbb{V}_{i-1}(\varphi, \mathfrak{J})\right\| \leq \sigma^{3}\left\|\mathbb{V}_{i-2}(\varphi, \mathfrak{J})\right\| \cdots \\
& \leq \sigma_{i+1}\left\|\mathbb{V}_{0}(\varphi, \mathfrak{J})\right\|
\end{aligned}
$$

For $i, n \in N$, we acquire

$$
\begin{aligned}
& \left\|C_{i}(\varphi, \mathfrak{\Im})-C_{n}(\varphi, \mathfrak{\Im})\right\|=\left\|\mathbb{V}_{i+n}(\varphi, \mathfrak{\Im})\right\| \\
& =\| C_{i}(\varphi, \mathfrak{J})-C_{i-1}(\varphi, \mathfrak{I})+\left(C_{i-1}(\varphi, \mathfrak{I})-C_{i-2}(\varphi, \mathfrak{I})\right) \\
& +\left(C_{i-2}(\varphi, \mathfrak{J})-C_{i-3}(\varphi, \mathfrak{I})\right)+\cdots+\left(C_{n+1}(\varphi, \mathfrak{J})-C_{n}(\varphi, \mathfrak{J})\right) \| \\
& \leq\left\|C_{i}(\varphi, \mathfrak{J})-C_{i-1}(\varphi, \mathfrak{J})\right\|+\left\|C_{i-1}(\varphi, \mathfrak{I})-C_{i-2}(\varphi, \mathfrak{J})\right\| \\
& +\left\|C_{i-2}(\varphi, \mathfrak{J})-C_{i-3}(\varphi, \mathfrak{J})\right\|+\cdots+\left\|C_{n+1}(\varphi, \mathfrak{I})-C_{n}(\varphi, \mathfrak{J})\right\| \\
& \leq \sigma^{i}\left\|\mathbb{V}_{0}(\varphi, \mathfrak{I})\right\|+\sigma^{i-1}\left\|\mathbb{V}_{0}(\varphi, \mathfrak{I})\right\|+\cdots+\sigma^{i+1}\left\|\mathbb{V}_{0}(\varphi, \mathfrak{I})\right\| \\
& =\left\|\mathbb{V}_{0}(\varphi, \mathfrak{\Im})\right\|\left(\sigma^{i}+\sigma^{i-1}+\sigma^{i+1}\right) \\
& =\left\|\mathbb{V}_{0}(\varphi, \mathfrak{I})\right\| \frac{1-\sigma^{i-n}}{1-\sigma^{i+1}} \sigma^{n+1} \text {. }
\end{aligned}
$$

Since $0<\sigma<1$, and $\mathbb{V}_{0}(\varphi, \mathfrak{I})$ is bounded, let us take $\beta$ $=1-\sigma /\left(1-\sigma_{i-n}\right) \sigma^{n+1}\left\|\mathbb{V}_{0}(\varphi, \mathfrak{I})\right\|$. Thus, $\left\{\mathbb{V}_{i}(\varphi, \mathfrak{\Im})\right\}_{i=0}^{\infty}$ forms a "Cauchy sequence" in $H$. It follows that the sequence $\left\{\mathbb{V}_{i}(\varphi, \mathfrak{J})\right\}_{i=0}^{\infty}$ is a convergent sequence with the $\operatorname{limit} \lim _{i \rightarrow \infty} \mathbb{V}{ }_{i}(\varphi, \mathfrak{\Im})=\mathbb{V}(\varphi, \mathfrak{\Im})$ for $\exists \mathbb{V}(\varphi, \mathfrak{\Im}) \in \mathscr{H}$. Hence, this ends the proof.

Theorem 9. Let $\sum_{h=0}^{k} \mathbb{V}_{h}(\varphi, \mathfrak{I})$ is finite and $\mathbb{V}(\varphi, \mathfrak{I})$ represents the obtained series solution. Let $\sigma>0$ such that $\| \mathbb{V}_{h+1}$ $(\varphi, \mathfrak{J})\|\leq\| \mathbb{V}_{h}(\varphi, \mathfrak{I}) \|$; then, the following relation gives the maximum absolute error [43].

$$
\left\|\mathbb{V}(\varphi, \mathfrak{\Im})-\sum_{h=0}^{k} \mathbb{V}_{h}(\varphi, \mathfrak{I})\right\|<\frac{\sigma^{k+1}}{1-\sigma}\left\|\mathbb{V}_{0}(\varphi, \mathfrak{I})\right\|
$$

Proof. Since $\sum_{h=0}^{k} \mathbb{V}_{h}(\varphi, \mathfrak{I})$ is finite, this implies that $\sum_{h=0}^{k}$ $\mathbb{V}_{h}(\varphi, \mathfrak{J})<\infty$.

Consider

$$
\begin{aligned}
\left\|\mathbb{V}(\varphi, \mathfrak{J})-\sum_{h=0}^{k} \mathbb{V}_{h}(\varphi, \mathfrak{I})\right\| & =\left\|\sum_{h=k+1}^{\infty} \mathbb{V}_{h}(\varphi, \mathfrak{I})\right\| \\
& \leq \sum_{h=k+1}^{\infty}\left\|\mathbb{V}_{h}(\varphi, \mathfrak{\Im})\right\| \\
& \leq \sum_{h=k+1}^{\infty} \sigma^{h}\left\|\mathbb{V}_{0}(\varphi, \mathfrak{\Im})\right\| \\
& \leq \sigma^{k+1}\left(1+\sigma+\sigma^{2}+\cdots\right)\left\|\mathbb{V}_{0}(\varphi, \mathfrak{I})\right\| \\
& \leq \frac{\sigma^{k+1}}{1-\sigma}\left\|\mathbb{V}_{0}(\varphi, \mathfrak{I})\right\| .
\end{aligned}
$$

This ends the theorem's proof.

\section{The General Procedure of NITM}

The general solution of fractional-order partial differential equation is as follows:

$$
\begin{gathered}
{ }^{\mathrm{CF}} D_{\mathfrak{J}}^{\wp} \mathbb{V}(\varphi, \mathfrak{I})+N \mathbb{V}(\varphi, \mathfrak{I})+M \mathbb{V}(\varphi, \mathfrak{J}) \\
=h(\varphi, \mathfrak{J}), i \in N, i-1<\wp \leq i,
\end{gathered}
$$

where $N$ is nonlinear and $M$ linear functions.

With the initial condition

$$
\mathbb{V}^{k}(\varphi, 0)=g_{k}(\varphi), k=0,1,2, \cdots, i-1,
$$

implementing the Yang transformation of Equation (30), we get

$$
\mathbb{Y}\left[D_{\mathfrak{I}}^{\wp} \mathbb{V}(\varphi, \mathfrak{I})\right]+\mathbb{Y}[N \mathbb{V}(\varphi, \mathfrak{I})+M \mathbb{V}(\varphi, \mathfrak{I})]=\mathbb{Y}[h(\varphi, \mathfrak{I})]
$$

Applying the Yang differentiation is given to

$$
\begin{aligned}
\mathbb{Y}[\mathbb{V}(\varphi, \mathfrak{J})]= & v \mathbb{V}(\varphi, 0)+(1+\wp(v-1)) \mathbb{Y}[h(\varphi, \mathfrak{J})] \\
& -(1+\wp(v-1)) \mathbb{Y}[N \mathbb{V}(\varphi, \mathfrak{J})+M \mathbb{V}(\varphi, \mathfrak{J})]
\end{aligned}
$$


Using inverse Yang transformation Equation (32), we get

$$
\begin{aligned}
\mathbb{V}(\varphi, \mathfrak{I})= & \mathbb{Y}^{-1}[\{v \mathbb{V}(\varphi, 0)+(1+\wp(v-1)) \mathbb{Y}[h(\varphi, \mathfrak{I})]\}] \\
& -\mathbb{Y}^{-1}[(1+\wp(v-1)) \mathbb{Y}[N \mathbb{V}(\varphi, \mathfrak{J})+M \mathbb{V}(\varphi, \mathfrak{J})]]
\end{aligned}
$$

By iterative method, we get

$$
\begin{aligned}
\mathbb{V}(\varphi, \mathfrak{I}) & =\sum_{i=0}^{\infty} \mathbb{V}_{i}(\varphi, \mathfrak{I}), \\
N\left(\sum_{i=0}^{\infty} \mathbb{V}_{i}(\varphi, \mathfrak{I})\right) & =\sum_{i=0}^{\infty} N\left[\mathbb{V}_{i}(\varphi, \mathfrak{\Im})\right] .
\end{aligned}
$$

The nonlinear term $N$ is identified as

$$
\begin{aligned}
N\left(\sum_{i=0}^{\infty} \mathbb{V}_{i}(\varphi, \mathfrak{I})\right)= & \mathbb{V}_{0}(\varphi, \mathfrak{I})+N\left(\sum_{i=0}^{\infty} \mathbb{V}_{i}(\varphi, \mathfrak{I})\right) \\
& -M\left(\sum_{i=0}^{\infty} \mathbb{V}_{i}(\varphi, \mathfrak{I})\right) .
\end{aligned}
$$

Putting Equations (35)-(37) in Equation (34), we have obtain the following solution:

$$
\begin{aligned}
& \sum_{i=0}^{\infty} \mathbb{V}_{i}(\varphi, \mathfrak{J})=\mathbb{Y}^{-1}\left[( 1 + \wp ( v - 1 ) ) \left(\sum_{i=0}^{\infty} s^{2-\varphi+i} u^{i}(\varphi, 0)\right.\right. \\
& +\mathbb{Y}[h(\varphi, \mathfrak{J})])]-\mathbb{Y}^{-1}[(1+\wp(v-1)) \mathbb{Y} \\
& \cdot\left[N\left(\sum_{i=0}^{\infty} \mathbb{V}_{i}(\varphi, \mathfrak{I})\right)-M\left(\sum_{i=0}^{\infty} \mathbb{V}_{i}(\varphi, \mathfrak{J})\right)\right], \\
& \mathbb{V}_{0}(\varphi, \mathfrak{\Im})=\mathbb{V}^{-1}[v \mathbb{V}(\varphi, 0)+(1+\wp(v-1)) \mathbb{Y}(g(\varphi, \mathfrak{I}))], \\
& \mathbb{V}_{1}(\varphi, \mathfrak{J})=-\mathbb{V}^{-1}\left[(1+\wp(v-1)) \mathbb{Y}\left[N\left[\mathbb{V}_{0}(\varphi, \mathfrak{I})\right]+M\left[\mathbb{V}_{0}(\varphi, \mathfrak{J})\right]\right]\right. \\
& \mathbb{V}_{m+1}(\varphi, \mathfrak{J})=-\mathbb{V}^{-1}\left[( 1 + \wp ( v - 1 ) ) \mathbb { Y } \left[-N\left(\sum_{i=0}^{i} \mathbb{V}_{i}(\varphi, \mathfrak{J})\right)\right.\right. \\
& \left.\left.-M\left(\sum_{i=0}^{i} \mathbb{V}_{i}(\varphi, \mathfrak{J})\right)\right]\right], m \geq 1 \text {. }
\end{aligned}
$$

Lastly, Equations (30) and (31) provide the $i$-term solution in series form which is expressed as

$$
\begin{aligned}
\mathbb{V}(\varphi, \mathfrak{I}) \cong & \mathbb{V}_{0}(\varphi, \mathfrak{I})+\mathbb{V}_{1}(\varphi, \mathfrak{I})+\mathbb{V}_{2}(\varphi, \mathfrak{I}) \\
& +\cdots . .,+\mathbb{V}_{i}(\varphi, \mathfrak{I}), i=1,2, \cdots
\end{aligned}
$$

Example 10. Consider fractional-order Cauchy-reaction diffusion equation as [44]

$$
{ }^{\mathrm{CF}} D_{\mathfrak{J}}^{\mathfrak{V}} \mathbb{V}(\varphi, \mathfrak{J})=D_{\mathfrak{J}}^{2} \mathbb{V}(\varphi, \mathfrak{I})-\mathbb{V}(\varphi, \mathfrak{I}), 0<\wp \leq 1,(\varphi, \mathfrak{I}) \in \Omega \subset R^{2}
$$

with initial and boundary conditions

$$
\begin{gathered}
\mathbb{V}(\varphi, 0)=e^{-\varphi}+\varphi=g(\varphi), \mathbb{V}(0, \mathfrak{I})=1=f_{0}(\mathfrak{I}), \\
\frac{\partial \mathbb{V}(0, \mathfrak{I})}{\partial \mathfrak{I}}=e^{-\mathfrak{I}}-1=f_{1}(\mathfrak{I}), \varphi, \mathfrak{I} \in R
\end{gathered}
$$

The methodology consists of applying Yang transformation first on both side in (40) and utilizing the differentiation property of Yang transformation, and we have

$$
\mathbb{Y}[\mathbb{V}(\varphi, \mathfrak{J})]=v\left(e^{-\varphi}+\varphi\right)+(1+\wp(v-1)) \mathbb{Y}\left[D_{\mathfrak{J}}^{2} \mathbb{V}-\mathbb{V}\right]
$$

Using Yang inverse transform, we get

$$
\mathbb{V}(\varphi, \mathfrak{J})=\left(e^{-\varphi}+\varphi\right)+\mathbb{Y}^{-1}\left((1+\wp(v-1)) \mathbb{Y}\left[D_{\Im}^{2} \mathbb{V}-\mathbb{V}\right]\right)
$$

Now, we apply the new iterative transform method

$$
\begin{aligned}
\mathbb{V}_{0}(\varphi, \mathfrak{I}) & =e^{-\varphi}+\varphi, \\
\mathbb{V}_{1}(\varphi, \mathfrak{I}) & =\mathbb{V}^{-1}\left[(1+\wp(v-1)) \mathbb{Y}\left\{D_{\mathfrak{I}}^{2} \mathbb{V}_{0}-\mathbb{V}_{0}\right\}\right]=-\varphi\{1+\wp \mathfrak{I}-\wp\}, \\
\mathbb{V}_{2}(\varphi, \mathfrak{I}) & =\mathbb{Y}^{-1}\left[(1+\wp(v-1)) \mathbb{Y}\left\{D_{\mathfrak{J}}^{2} \mathbb{V}_{1}-\mathbb{V}_{1}\right\}\right] \\
& =\varphi\left\{(1-\wp) 2 \wp \mathfrak{I}+(1-\wp)^{2}+\frac{\wp^{2} \mathfrak{I}^{2}}{2}\right\}, \\
\mathbb{V}_{3}(\varphi, \mathfrak{I}) & =\mathbb{Y}^{-1}\left[(1+\wp(v-1)) \mathbb{Y}\left\{D_{\mathfrak{J}^{2}}^{2} \mathbb{V}_{2}-\mathbb{V}_{2}\right\}\right] \\
& =-\varphi\left\{(1-\wp)^{2} 3 \wp \mathfrak{I}+(1-\wp)^{3}+\frac{3 \wp^{2}(1-\wp) \mathfrak{J}^{2}}{2}+\frac{\wp^{3} \mathfrak{I}^{3}}{3 !}\right\} \\
& \vdots
\end{aligned}
$$

The series type solution is given as

$$
\begin{aligned}
\mathbb{V}(\varphi, \mathfrak{I})= & \mathbb{V}_{0}(\varphi, \mathfrak{I})+\mathbb{V}_{1}(\varphi, \mathfrak{I})+\mathbb{V}_{2}(\varphi, \mathfrak{I})+\mathbb{V}_{3}(\varphi, \mathfrak{I}) \\
& +\cdots \mathbb{V}_{i}(\varphi, \mathfrak{I}) .
\end{aligned}
$$


The approximate solution is achieved as

$$
\begin{aligned}
\mathbb{V}(\varphi, \mathfrak{I})= & e^{-\varphi}+\varphi\{1-\{1+\wp \mathfrak{\Im}-\wp\} \\
& +\left\{(1-\wp) 2 \wp \mathfrak{I}+(1-\wp)^{2}+\frac{\wp^{2} \mathfrak{J}^{2}}{2}\right\} \\
& -\left\{(1-\wp)^{2} 3 \wp \mathfrak{I}+(1-\wp)^{3}+\frac{3 \wp^{2}(1-\wp) \mathfrak{J}^{2}}{2}\right. \\
& \left.\left.+\frac{\wp^{3} \mathfrak{J}^{3}}{3 !}\right\}+\cdots\right\} .
\end{aligned}
$$

Now applying the HPTM, we get

$$
\begin{aligned}
\sum_{i=0}^{\infty} p^{i} \mathbb{V}_{i}(\varphi, \mathfrak{I})= & \left(e^{-\varphi}+\varphi\right)+p \\
& \cdot\left\{\mathbb{Y}^{-1}\left((1+\wp(v-1)) \mathbb{Y}\left[\sum_{i=0}^{\infty} p^{i} H_{i}(\mathbb{V})\right]\right)\right\},
\end{aligned}
$$

where the polynomials represent the nonlinear functions are $H_{i}(\mathbb{V})$. For instance, the terms of He's polynomials are achieved through the recursive relationship $H_{i}(\mathbb{V})=D_{\mathfrak{J}}^{2} \mathbb{V}_{i}$ $-\mathbb{V}_{i}, \forall n \in N$. Now, as the correspond power coefficients of $p$ is comparison on both sides, the following solution is obtained as follows:

$$
\begin{aligned}
p^{0}: \mathbb{V}_{0}(\varphi, \mathfrak{I}) & =e^{-\varphi}+\varphi, \\
p^{1}: \mathbb{V}_{1}(\varphi, \mathfrak{I}) & =\left[\mathbb{Y}^{-1}\left\{(1+\wp(v-1)) \mathbb{Y}\left(H_{0}(\mathbb{V})\right)\right\}\right]=-\varphi\{1+\wp \mathfrak{I}-\wp\}, \\
p^{2}: \mathbb{V}_{2}(\varphi, \mathfrak{I}) & =\left[\mathbb{Y}^{-1}\left\{(1+\wp(v-1)) \mathbb{Y}\left(H_{1}(\mathbb{V})\right)\right\}\right] \\
& =\varphi\left\{(1-\wp) 2 \wp \mathfrak{I}+(1-\wp)^{2}+\frac{\wp^{2} \mathfrak{J}^{2}}{2}\right\},
\end{aligned}
$$$$
p^{3}: \mathbb{V}_{3}(\varphi, \mathfrak{I})=\left[\mathbb{V}^{-1}\left\{(1+\wp(v-1)) \mathbb{Y}\left(H_{2}(\mathbb{V})\right)\right\}\right]
$$$$
=-\varphi\left\{(1-\wp)^{2} 3 \wp \mathfrak{I}+(1-\wp)^{3}\right.
$$$$
\left.+\frac{3 \wp^{2}(1-\wp) \mathfrak{\Im}^{2}}{2}+\frac{\wp^{3} \mathfrak{\Im}^{3}}{3 !}\right\}
$$

Then, the homotopy perturbation method series form solution is defined as

$$
\mathbb{V}(\varphi, \mathfrak{J})=\sum_{i=0}^{\infty} p^{i} \mathbb{V}_{i}(\varphi, \mathfrak{J})
$$

The analytical result of the above equation is defined as

$$
\begin{aligned}
\mathbb{V}(\varphi, \mathfrak{I})= & e^{-\varphi}+\varphi\{1-\{1+\wp \mathfrak{I}-\wp\} \\
& +\left\{(1-\wp) 2 \wp \mathfrak{I}+(1-\wp)^{2}+\frac{\wp^{2} \mathfrak{J}^{2}}{2}\right\} \\
& -\left\{(1-\wp)^{2} 3 \wp \mathfrak{I}+(1-\wp)^{3}\right. \\
& \left.\left.+\frac{3 \wp^{2}(1-\wp) \mathfrak{I}^{2}}{2}+\frac{\wp^{3} \mathfrak{J}^{3}}{3 !}\right\}+\cdots\right\}=\varphi \sum_{i=0}^{\infty} \frac{\left(\mathfrak{J}^{\mathfrak{p}}\right)^{i}}{\Gamma(i \wp+1)}, \\
\mathbb{V}(\varphi, \mathfrak{J})= & e^{-\varphi}+\varphi E_{\wp}\left(\mathfrak{J}^{\wp}\right) .
\end{aligned}
$$

The exact result of the above equation is

$$
\mathbb{V}(\varphi, \mathfrak{\Im})=e^{-\varphi}+\varphi e^{-\mathfrak{I}}
$$

Figure 1 shows the analytical solution of two methods at different fractional-order $\wp=1$ and 0.8 , and Figure 2 shows separate fractional-order at $\wp=0.6$ and 0.4 with close contact with each other. In Figure 3, the graph shows the different fractional-order $\wp$ of Example 10.

Example 11. Consider fractional-order Cauchy-reaction diffusion equation as [44]

$$
\begin{aligned}
{ }^{\mathrm{CF}} D_{\mathfrak{J}} \mathbb{V}(\varphi, \mathfrak{I}) & =D_{\mathfrak{J}}^{2} \mathbb{V}(\varphi, \mathfrak{I})-\left(1+4 \varphi^{2}\right) \mathbb{V}(\varphi, \mathfrak{I}), 0<\wp \leq 1,(\varphi, t) \\
& \in \Omega \subset R^{2},
\end{aligned}
$$

with initial condition

$$
\mathbb{V}(\varphi, 0)=e^{\varphi^{2}}
$$

and the exact result is given as

$$
\mathbb{V}(\varphi, \mathfrak{\Im})=e^{\varphi^{2}+1}
$$

Now, we apply the new iterative transform method

$$
\begin{aligned}
\mathbb{V}_{0}(\varphi, \mathfrak{I})= & e^{\varphi^{2}}, \\
\mathbb{V}_{1}(\varphi, \mathfrak{I})= & \mathbb{V}^{-1}\left[( 1 + \wp ( v - 1 ) ) \mathbb { Y } \left\{D_{\mathfrak{J}}^{2} \mathbb{V}_{0}(\varphi, \mathfrak{I})\right.\right. \\
& \left.\left.-\left(1+4 \varphi^{2}\right) \mathbb{V}_{0}(\varphi, \mathfrak{I})\right\}\right]=e^{\varphi^{2}}\{1+\wp \mathfrak{I}-\wp\}, \\
\mathbb{V}_{2}(\varphi, \mathfrak{I})= & \mathbb{V}^{-1}\left[(1+\wp(v-1)) \mathbb{Y}\left\{D_{\mathfrak{J}}^{2} \mathbb{V}_{1}(\varphi, \mathfrak{I})-\left(1+4 \varphi^{2}\right) \mathbb{V}_{1}(\varphi, \mathfrak{I})\right\}\right] \\
= & e^{\varphi^{2}}\left\{(1-\wp) 2 \wp \mathfrak{I}+(1-\wp)^{2}+\frac{\wp^{2} \mathfrak{I}^{2}}{2}\right\}, \\
\mathbb{V}_{3}(\varphi, \mathfrak{I})= & \mathbb{Y}^{-1}\left[(1+\wp(v-1)) \mathbb{Y}\left\{D_{\mathfrak{J}}^{2} \mathbb{V}_{2}(\varphi, \mathfrak{I})-\left(1+4 \varphi^{2}\right) \mathbb{V}_{2}(\varphi, \mathfrak{I})\right\}\right] \\
= & e^{\varphi^{2}}\left\{(1-\wp)^{2} 3 \wp \mathfrak{I}+(1-\wp)^{3}+\frac{3 \wp^{2}(1-\wp) \mathfrak{J}^{2}}{2}+\frac{\wp^{3} \mathfrak{I}^{3}}{3 !}\right\},
\end{aligned}
$$



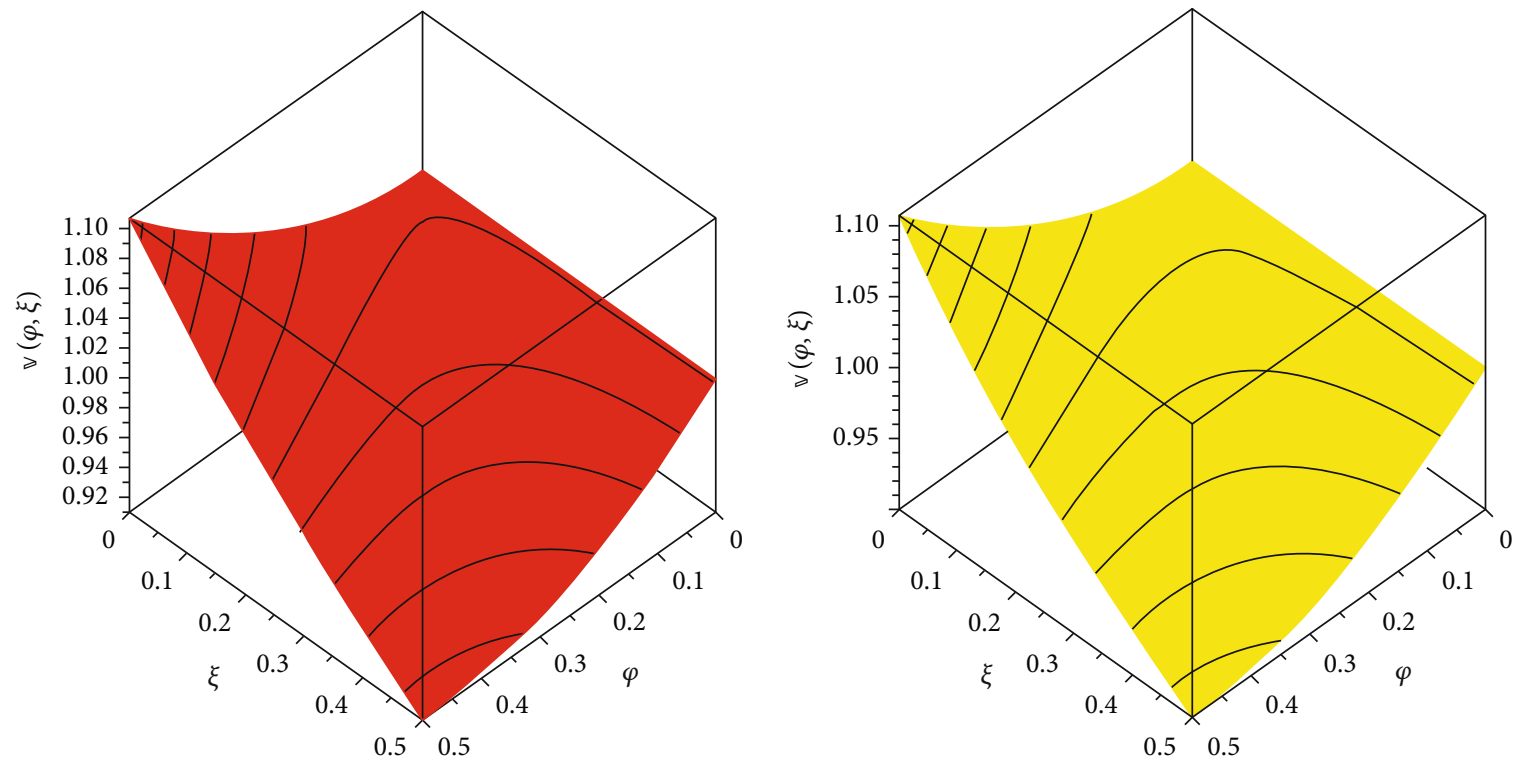

Figure 1: (a) $\wp=1$ and (b) the fractional-order $\wp=0.8$ of Example 10 .
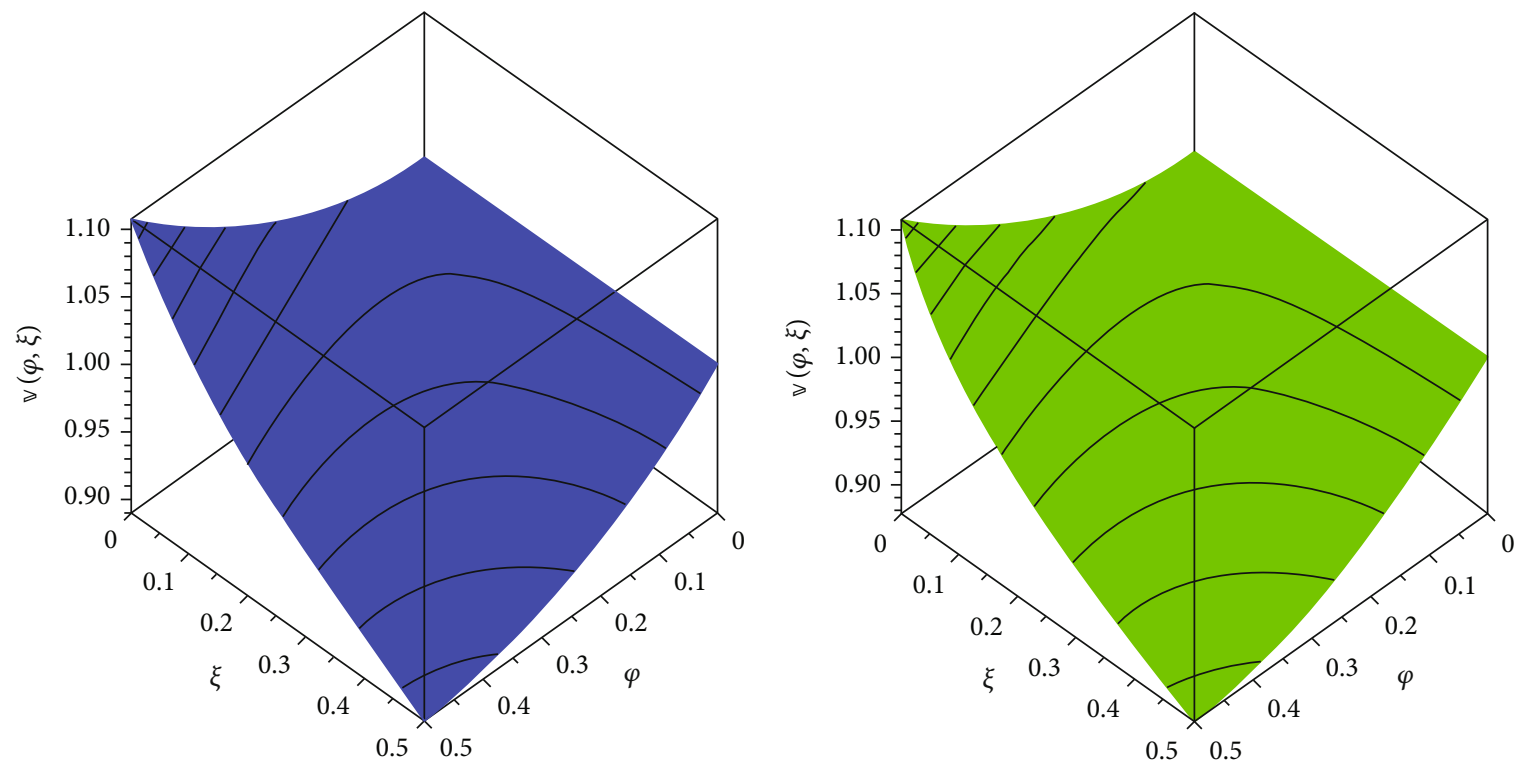

FIGURE 2: Different fractional-order of $\wp=0.6$ and 0.4 of Example 10.

The series type solution is given as

$\mathbb{V}(\varphi, \mathfrak{J})=\mathbb{V}_{0}(\varphi, \mathfrak{J})+\mathbb{V}_{1}(\varphi, \mathfrak{J})+\mathbb{V}_{2}(\varphi, \mathfrak{I})+\mathbb{V}_{3}(\varphi, \mathfrak{J})+\cdots \mathbb{V}_{i}(\varphi, \mathfrak{J})$

The approximate solution of the above equation is defined as

$$
\begin{aligned}
\mathbb{V}(\varphi, \mathfrak{I})= & e^{\varphi^{2}}\left\{1+\{1+\wp \mathfrak{I}-\wp\}+\left\{(1-\wp) 2 \wp \mathfrak{I}+(1-\wp)^{2}+\frac{\wp^{2} \mathfrak{J}^{2}}{2}\right\}\right. \\
& \left.+\left\{(1-\wp)^{2} 3 \wp \mathfrak{I}+(1-\wp)^{3}+\frac{3 \wp^{2}(1-\wp) \mathfrak{J}^{2}}{2}+\frac{\wp^{3} \mathfrak{J}^{3}}{3 !}\right\}+\cdots\right\},
\end{aligned}
$$

$$
\mathbb{V}(\varphi, \mathfrak{J})=e^{\varphi^{2}} E_{\wp}\left(\mathfrak{J}^{\wp}\right)
$$

Now by applying homotopy perturbation transform method, we get

$$
\begin{aligned}
& \sum_{i=0}^{\infty} p^{i} \mathbb{V}_{i}(\varphi, \mathfrak{I}) \\
& \quad=e^{\varphi^{2}}+p\left\{\mathbb{Y}^{-1}\left((1+\wp(v-1)) \mathbb{Y}\left[\sum_{i=0}^{\infty} p^{i} H_{i}(w)\right]\right)\right\} .
\end{aligned}
$$




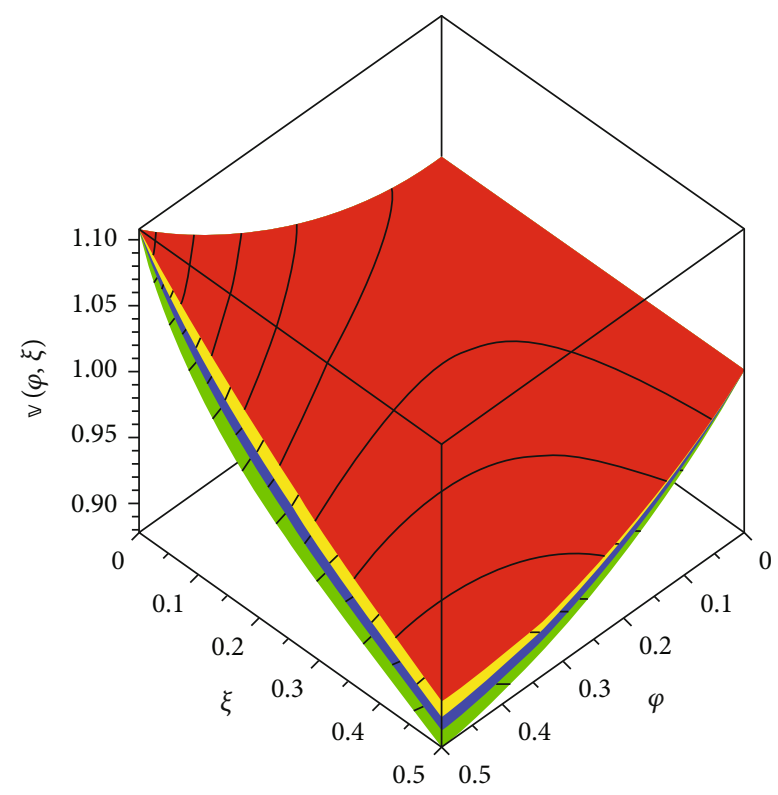

FIgURE 3: The different fractional-order $\wp$ of Example 10 .

Comparing the coefficients of power $p$, we get

$$
\begin{aligned}
p^{0}: \mathbb{V}_{0}(\varphi, \mathfrak{I}) & =e^{\varphi^{2}}, \\
p^{1}: \mathbb{V}_{1}(\varphi, \mathfrak{I}) & =\left\{\mathbb{Y}^{-1}\left((1+\wp(v-1)) \mathbb{Y}\left[H_{0}(w)\right]\right)\right\}=e^{\varphi^{2}}\{1+\wp \mathfrak{I}-\wp\}, \\
p^{2}: \mathbb{V}_{2}(\varphi, \mathfrak{I}) & =\left\{\mathbb{V}^{-1}\left((1+\wp(v-1)) \mathbb{Y}\left[H_{1}(w)\right]\right)\right\} \\
& =e^{\varphi^{2}}\left\{(1-\wp) 2 \wp \mathfrak{I}+(1-\wp)^{2}+\frac{\wp^{2} \mathfrak{J}^{2}}{2}\right\}, \\
p^{3}: \mathbb{V}_{3}(\varphi, \mathfrak{I}) & =\left\{\mathbb{Y}^{-1}\left((1+\wp(v-1)) \mathbb{Y}\left[H_{2}(w)\right]\right)\right\} \\
& =e^{\varphi^{\varphi^{2}}}\left\{(1-\wp)^{2} 3 \wp \mathfrak{I}+(1-\wp)^{3}+\frac{3 \wp^{2}(1-\wp) \mathfrak{J}^{2}}{2}+\frac{\wp^{3} \mathfrak{J}^{3}}{3 !}\right\},
\end{aligned}
$$

The HPTM series solution is given as

$$
\begin{aligned}
\mathbb{V}(\varphi, \mathfrak{I})= & \sum_{i=0}^{\infty} p^{i} \mathbb{V}_{i}(\varphi, \mathfrak{I}), \\
\mathbb{V}(\varphi, \mathfrak{I})= & e^{\varphi^{2}}\{1+\{1+\wp \mathfrak{I}-\wp\} \\
& +\left\{(1-\wp) 2 \wp \mathfrak{I}+(1-\wp)^{2}+\frac{\wp^{2} \mathfrak{J}^{2}}{2}\right\} \\
& +\left\{(1-\wp)^{2} 3 \wp \mathfrak{I}+(1-\wp)^{3}+\frac{3 \wp^{2}(1-\wp) \mathfrak{J}^{2}}{2}\right. \\
& \left.\left.+\frac{\wp^{3} \mathfrak{J}^{3}}{3 !}\right\}+\cdots\right\},
\end{aligned}
$$$$
\mathbb{V}(\varphi, \mathfrak{J})=e^{\varphi^{2}} E_{\mathfrak{\wp}}\left(\mathfrak{J}^{\mathfrak{l}}\right)
$$

Now $\wp=1$; then, the actual result of Equation (52) is $\mathbb{V}$ $(\varphi, \mathfrak{I})=e^{\varphi^{2}+\mathfrak{I}}$.

Figure 4 shows the analytical solution of two methods at different fractional-order $\wp=1$ and 0.8 , and Figure 5 shows the separate fractional-order at $\wp=0.6$ and 0.4 with close contact with each other. In Figure 6, the graph shows the different fractional-order $\wp$ of Example 11.

Example 12. Consider fractional-order Cauchy-reaction diffusion equation [44]

${ }^{\mathrm{CF}} D_{\widetilde{J}}^{\mathfrak{p}} \mathbb{V}(\varphi, \mathfrak{I})=D_{\mathfrak{J}}^{2} \mathbb{V}(\varphi, \mathfrak{\Im})+2 \mathfrak{J} \mathbb{V}(\varphi, \mathfrak{\Im}), 0<\wp \leq 1,(\varphi, \mathfrak{J}) \in \Omega \subset R^{2}$,

with initial condition

$$
\mathbb{V}(\varphi, 0)=e^{\varphi}
$$

The exact result is

$$
\mathbb{V}(\varphi, \mathfrak{I})=e^{\varphi+\Im+\Im^{2}}
$$

By using the Yang transformation, we get

$\mathbb{V}(\varphi, \mathfrak{I})=\left(e^{\varphi}\right)+\mathbb{Y}^{-1}\left[(1+\wp(v-1)) \mathbb{Y}\left(D_{\mathfrak{J}}^{2} \mathbb{V}(\varphi, \mathfrak{I})+2 \mathfrak{I} \mathbb{V}(\varphi, \mathfrak{I})\right)\right]$

Now, we apply the new iterative transform method

$$
\begin{aligned}
\mathbb{V}_{0}(\varphi, \mathfrak{I})= & e^{\varphi}, \\
\mathbb{V}_{1}(\varphi, \mathfrak{I})= & \mathbb{Y}^{-1}\left[(1+\wp(v-1)) \mathbb{Y}\left\{D_{\mathfrak{J}}^{2} \mathbb{V}_{0}(\varphi, \mathfrak{I})+2 \mathfrak{J} \mathbb{V}_{0}(\varphi, \mathfrak{I})\right\}\right] \\
= & e^{\varphi}\left(\{1+\wp \mathfrak{I}-\wp\}+\left\{(1-\wp) 2 \wp \mathfrak{I}+(1-\wp)^{2}+\frac{\wp^{2} \mathfrak{J}^{2}}{2}\right\}\right), \\
\mathbb{V}_{2}(\varphi, \mathfrak{I})= & \mathbb{V}^{-1}\left[(1+\wp(v-1)) \mathbb{Y}\left\{D_{\mathfrak{J}}^{2} \mathbb{V}_{1}(\varphi, \mathfrak{I})+2 \mathfrak{I} \mathbb{V}_{1}(\varphi, \mathfrak{I})\right\}\right] \\
= & e^{\varphi}\left(\left\{(1-\wp) 2 \wp \mathfrak{I}+(1-\wp)^{2}+\frac{\wp^{2} \mathfrak{J}^{2}}{2}\right\}\right. \\
& \left.+\left\{(1-\wp)^{2} 3 \wp \mathfrak{I}+(1-\wp)^{3}+\frac{3 \wp^{2}(1-\wp) \mathfrak{J}^{2}}{2}+\frac{\wp^{3} \mathfrak{J}^{3}}{3 !}\right\}\right), \\
& \vdots
\end{aligned}
$$

The series type solution is given as

$\mathbb{V}(\varphi, \mathfrak{J})=\mathbb{V}_{0}(\varphi, \mathfrak{I})+\mathbb{V}_{1}(\varphi, \mathfrak{I})+\mathbb{V}_{2}(\varphi, \mathfrak{J})+\mathbb{V}_{3}(\varphi, \mathfrak{I})+\cdots \mathbb{V}_{i}(\varphi, \mathfrak{J})$ 

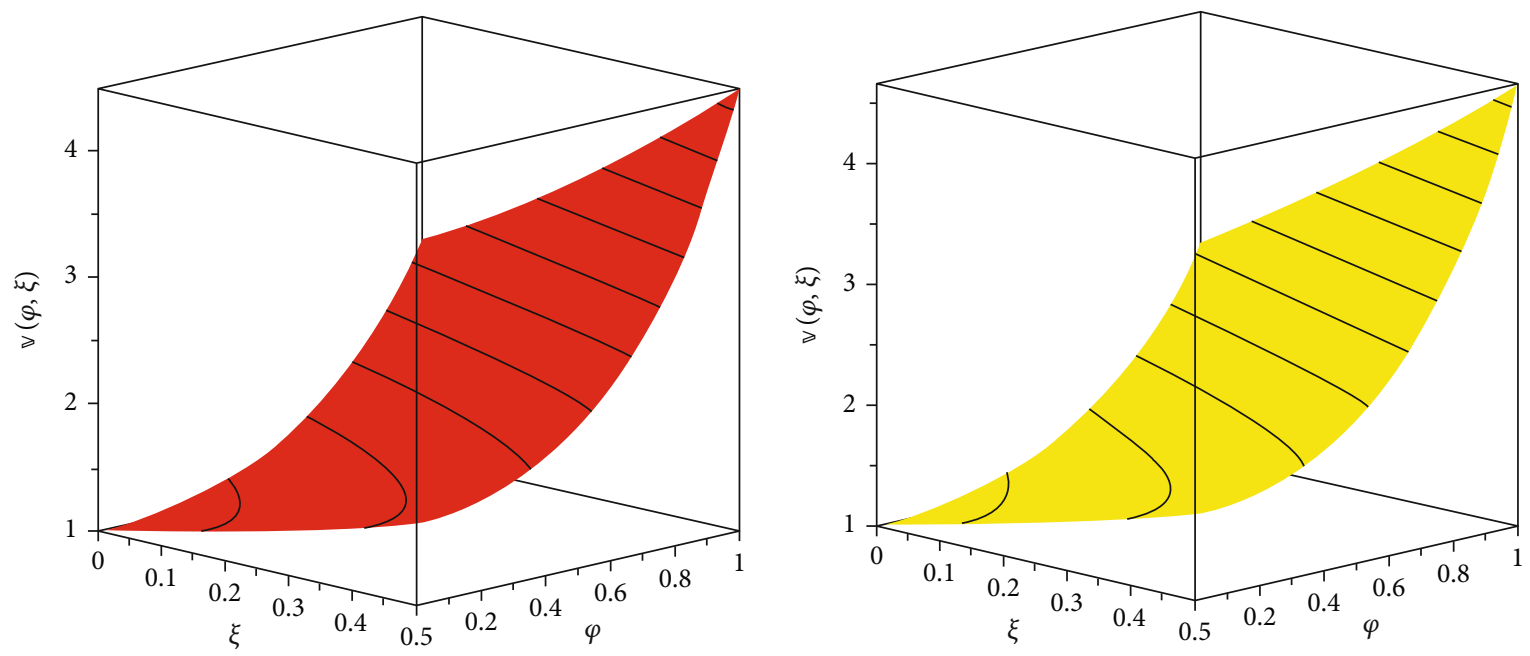

Figure 4: (a) $\wp=1$ and (b) the fractional-order $\wp=0.8$ of Example 10 .
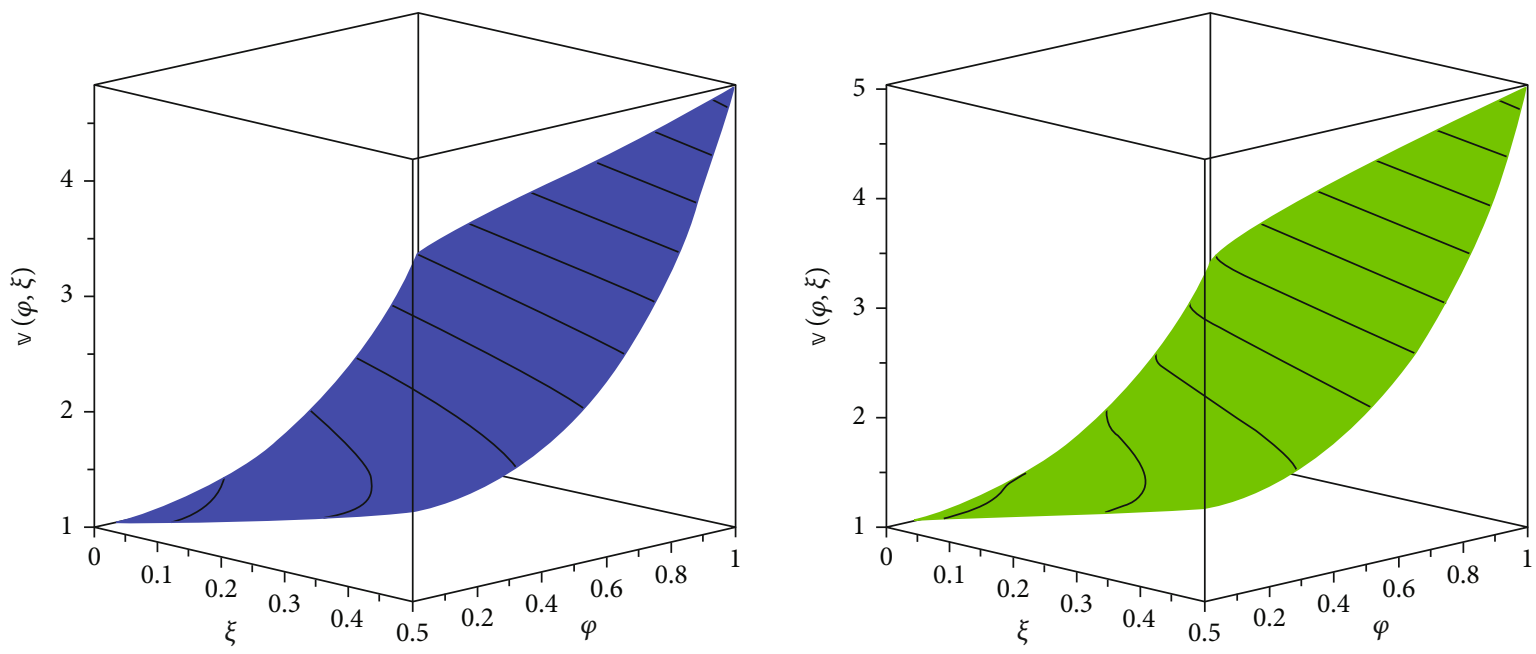

Figure 5: The different fractional-order of $\wp=0.6$ and 0.4 of Example 10.

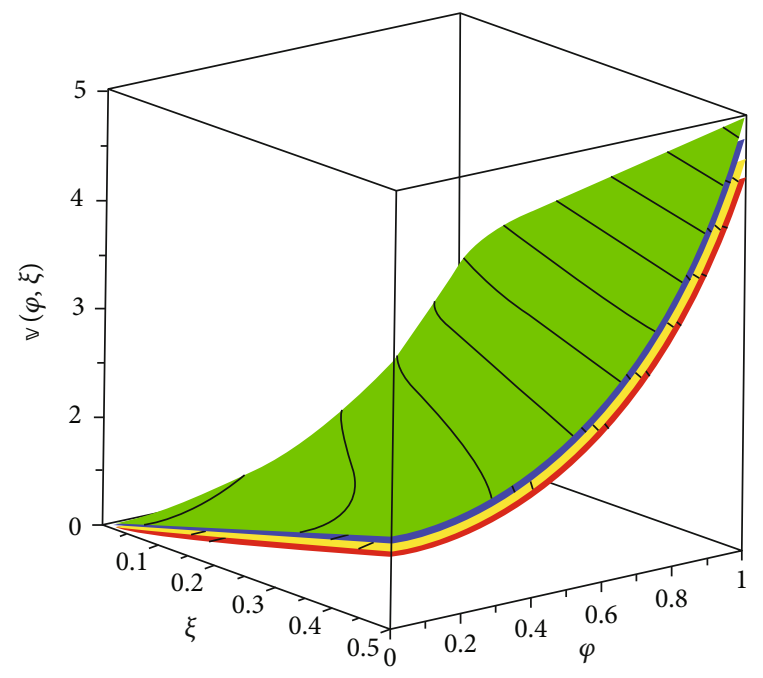

The approximate solution of the above equation is defined as

$$
\begin{aligned}
\mathbb{V}(\varphi, \mathfrak{I})= & e^{\varphi}+e^{\varphi}\left(\{1+\wp \mathfrak{I}-\wp\}+\left\{(1-\wp) 2 \wp \mathfrak{I}+(1-\wp)^{2}+\frac{\wp^{2} \mathfrak{J}^{2}}{2}\right\}\right. \\
& +e^{\varphi}\left(\left\{(1-\wp) 2 \wp \mathfrak{I}+(1-\wp)^{2}+\frac{\wp^{2} \mathfrak{J}^{2}}{2}\right\}\right. \\
& \left.+\left\{(1-\wp)^{2} 3 \wp \mathfrak{I}+(1-\wp)^{3}+\frac{3 \wp^{2}(1-\wp) \mathfrak{I}^{2}}{2}+\frac{\wp^{3} \mathfrak{I}^{3}}{3 !}\right\}\right)+
\end{aligned}
$$

Now, using HPM, we get

$$
\sum_{i=0}^{\infty} p^{i} \mathbb{V}_{i}(\varphi, \mathfrak{J})=e^{\varphi}+p\left[\mathbb{Y}^{-1}\left\{(1+\wp(v-1)) \mathbb{Y}\left(\sum_{i=0}^{\infty} p^{i} H_{i}(w)\right)\right\}\right]
$$

FIGURE 6: The different fractional-order $\wp$ of Example 10. 

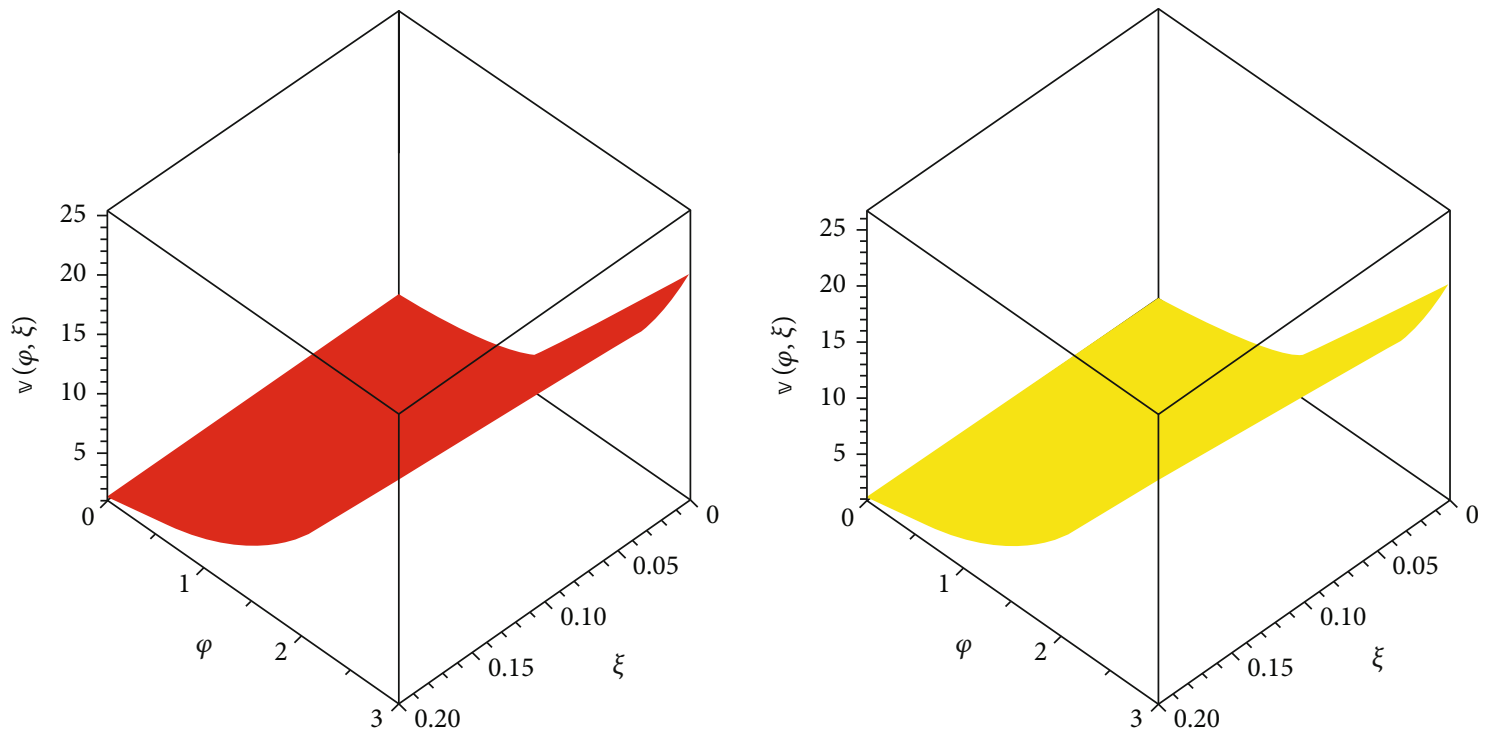

Figure 7: (a) $\wp=1$ and (b) the fractional-order $\wp=0.8$ of Example 10 .

Comparing the coefficients of power $p$, we get

$p^{0}: \mathbb{V}_{0}(\varphi, \mathfrak{\Im})=e^{\varphi}$,

$$
\begin{aligned}
& p^{1}: \mathbb{V}_{1}(\varphi, \mathfrak{J})=\left[\mathbb{Y}^{-1}\left\{(1+\wp(v-1)) \mathbb{Y}\left(H_{0}(w)\right)\right\}\right] \\
& =e^{\varphi}\left(\{1+\wp \mathfrak{I}-\wp\}+\left\{(1-\wp) 2 \wp \mathfrak{I}+(1-\wp)^{2}+\frac{\wp^{2} \mathfrak{I}^{2}}{2}\right\}\right), \\
& p^{2}: \mathbb{V}_{2}(\varphi, \mathfrak{J})=\left[\mathbb{Y}^{-1}\left\{(1+\mathscr{\wp}(v-1)) \mathbb{Y}\left(H_{1}(w)\right)\right\}\right] \\
& =e^{\varphi}\left(\left\{(1-\wp) 2 \wp \mathfrak{I}+(1-\wp)^{2}+\frac{\wp^{2} \mathfrak{J}^{2}}{2}\right\}\right. \\
& \left.+\left\{(1-\wp)^{2} 3 \wp \mathfrak{I}+(1-\wp)^{3}+\frac{3 \wp^{2}(1-\wp) \mathfrak{J}^{2}}{2}+\frac{\wp^{3} \mathfrak{J}^{3}}{3 !}\right\}\right) .
\end{aligned}
$$

Proceeding in this path, the rest of the $\mathbb{V} n(\varphi, \mathfrak{I})$ for $n \geq 3$ component can be completely recovered and the series solution can therefore be absolutely determined. Eventually, we approximate the numerical solution $\mathbb{V}(\varphi, \mathfrak{J})$ to the truncated series.

$$
\begin{aligned}
\mathbb{V}(\varphi, \mathfrak{I})= & \lim _{N \longrightarrow \infty} \sum_{n=1}^{N} \mathbb{V}_{i}(\varphi, \mathfrak{I}) \\
\mathbb{V}(\varphi, \mathfrak{I})= & e^{\varphi}+e^{\varphi}\left(\{1+\wp \mathfrak{I}-\wp\}+\left\{(1-\wp) 2 \wp \mathfrak{I}+(1-\wp)^{2}+\frac{\wp^{2} \mathfrak{J}^{2}}{2}\right\}\right) \\
& +e^{\varphi}\left(\left\{(1-\wp) 2 \wp \mathfrak{I}+(1-\wp)^{2}+\frac{\wp^{2} \mathfrak{J}^{2}}{2}\right\}\right. \\
& \left.+\left\{(1-\wp)^{2} 3 \wp \mathfrak{I}+(1-\wp)^{3}+\frac{3 \wp^{2}(1-\wp) \mathfrak{I}^{2}}{2}+\frac{\wp^{3} \mathfrak{J}^{3}}{3 !}\right\}\right)+\cdots
\end{aligned}
$$

Now for $\wp=1$, the closed form of the above series is

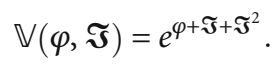

Figure 7 shows the analytical solution of two methods at different fractional-order $\wp=1$ and 0.8 , and Figure 8 shows the separate fractional-order at $\wp=0.6$ and 0.4 with close contact with each other. In Figure 9, the graph shows the different fractional-order $\wp$ of Example 12.

Example 13. Consider fractional-order Cauchy-reaction diffusion equation as [44]

$$
\begin{aligned}
{ }^{\mathrm{CF}} D_{\mathfrak{J}}^{\mathfrak{S}} \mathbb{V}(\varphi, \mathfrak{I})= & D_{\mathfrak{J}}^{2} \mathbb{V}(\varphi, \mathfrak{I})-\left(4 \varphi^{2}-2 \mathfrak{I}+2\right) \mathbb{V} \\
& \cdot(\varphi, \mathfrak{I}), 0<\wp \leq 1,(\varphi, \mathfrak{I}) \in \Omega \subset R^{2},
\end{aligned}
$$

with initial condition

$$
\mathbb{V}(\varphi, 0)=e^{\varphi^{2}}
$$

The exact result is

$$
\mathbb{V}(\varphi, \mathfrak{J})=e^{\varphi^{2}+\mathfrak{\Im}^{2}}
$$

Now, we apply the new iterative transform method

$$
\begin{aligned}
\mathbb{V}_{0}(\varphi, \mathfrak{I})= & e^{\varphi^{2}}, \\
\mathbb{V}_{1}(\varphi, \mathfrak{I})= & \mathbb{Y}^{-1}\left[( 1 + \wp ( v - 1 ) ) \mathbb { Y } \left\{D_{\mathfrak{J}}^{2} \mathbb{V}_{0}(\varphi, \mathfrak{I})\right.\right. \\
& \left.\left.-\left(4 \varphi^{2}-2 \mathfrak{I}+2\right) \mathbb{V}_{0}(\varphi, \mathfrak{I})\right\}\right]=e^{\varphi^{2}}\{1+\wp \mathfrak{I}-\wp\}, \\
\mathbb{V}_{2}(\varphi, \mathfrak{I})= & \mathbb{V}^{-1}\left[( 1 + \wp ( v - 1 ) ) \mathbb { Y } \left\{D_{\mathfrak{J}}^{2} \mathbb{V}_{1}(\varphi, \mathfrak{I})\right.\right. \\
& \left.\left.-\left(4 \varphi^{2}-2 \mathfrak{I}+2\right) \mathbb{V}_{1}(\varphi, \mathfrak{J})\right\}\right] \\
= & e^{\varphi^{2}}\left\{(1-\wp) 2 \wp \mathfrak{I}+(1-\wp)^{2}+\frac{\wp^{2} \mathfrak{J}^{2}}{2}\right\},
\end{aligned}
$$



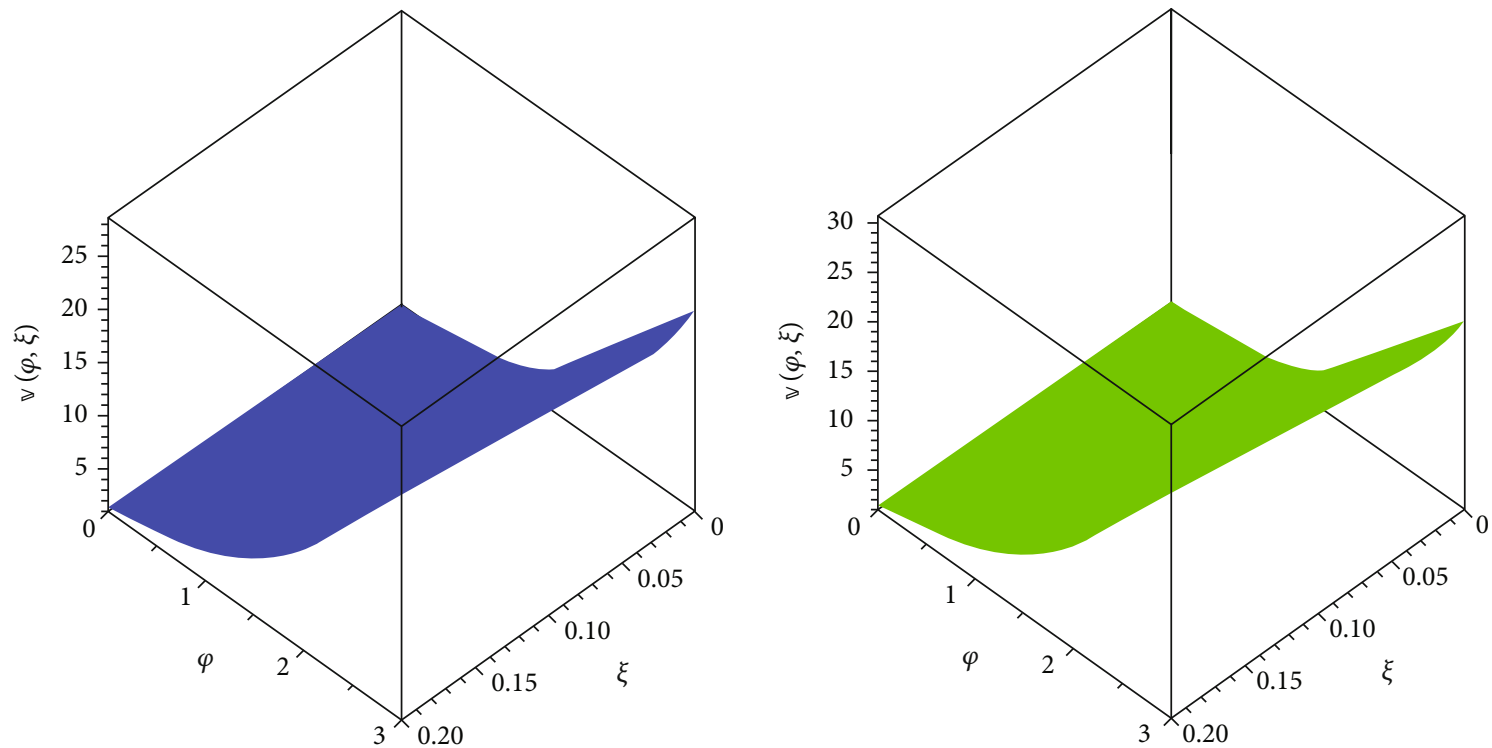

Figure 8: The different fractional-order of $\wp=0.6$ and 0.4 of Example 10.
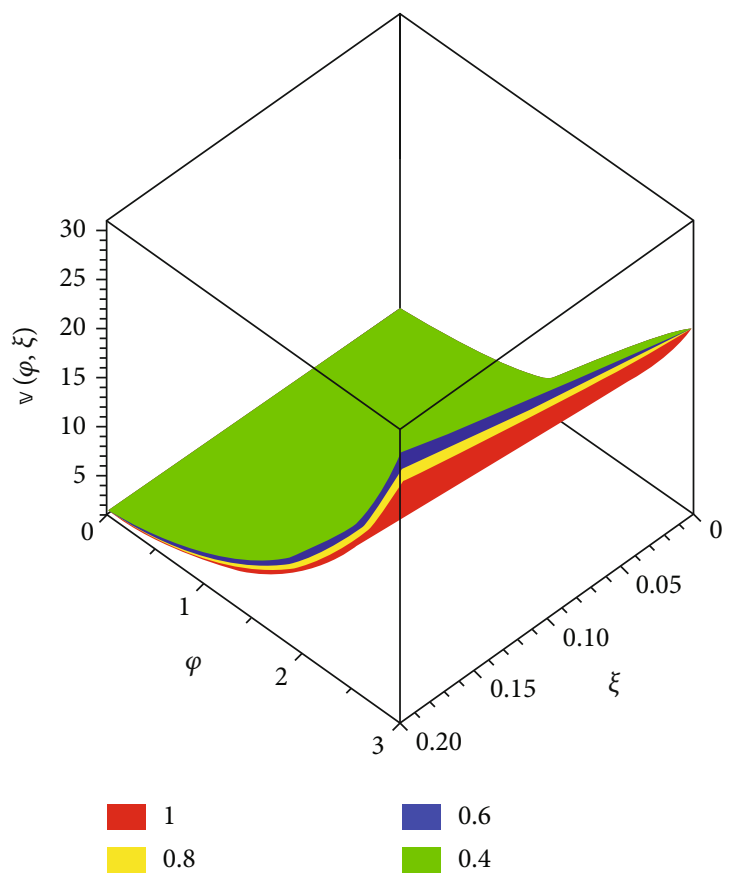

FIgURE 9: The different fractional-order $\wp$ of Example 10.

$$
\begin{aligned}
\mathbb{V}_{3}(\varphi, \mathfrak{I})= & \mathbb{V}^{-1}\left[( 1 + \wp ( \nu - 1 ) ) \mathbb { Y } \left\{D_{\mathfrak{J}}^{2} \mathbb{V}_{2}(\varphi, \mathfrak{I})\right.\right. \\
& \left.\left.-\left(4 \varphi^{2}-2 \mathfrak{I}+2\right) \mathbb{V}_{2}(\varphi, \mathfrak{I})\right\}\right] \\
= & e^{\varphi^{2}}\left\{(1-\wp)^{2} 3 \wp \mathfrak{I}+(1-\wp)^{3}+\frac{3 \wp^{2}(1-\wp) \mathfrak{J}^{2}}{2}+\frac{\wp^{3} \mathfrak{J}^{3}}{3 !}\right\}
\end{aligned}
$$$$
\vdots
$$

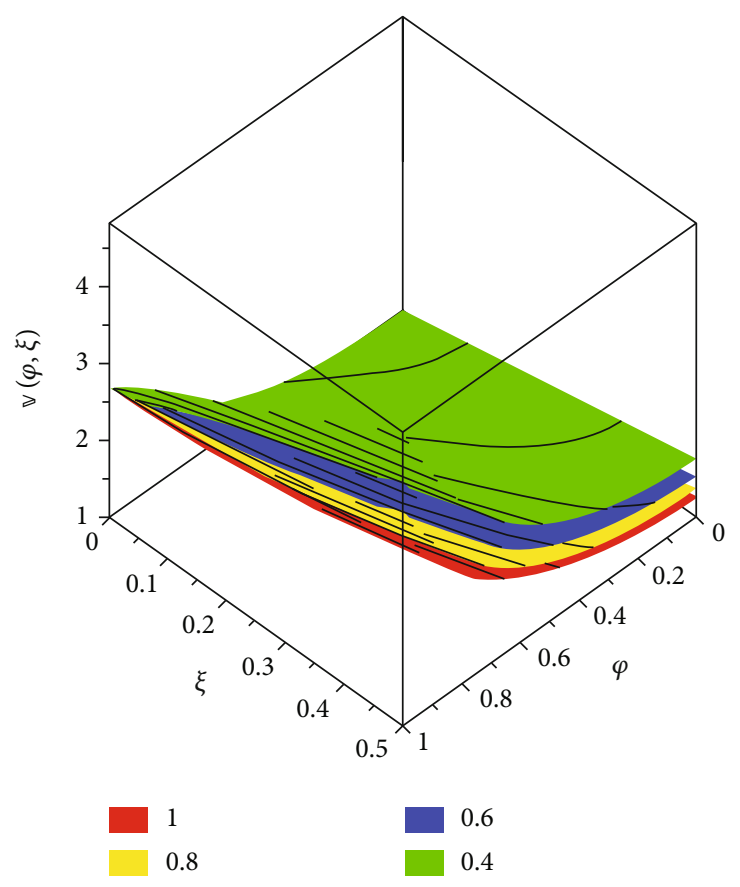

FIgURE 10: The different fractional-order $\wp$ of Example 13.

The series type solution is given as

$$
\begin{aligned}
\mathbb{V}(\varphi, \mathfrak{I})= & \mathbb{V}_{0}(\varphi, \mathfrak{I})+\mathbb{V}_{1}(\varphi, \mathfrak{I})+\mathbb{V}_{2}(\varphi, \mathfrak{I}) \\
& +\mathbb{V}_{3}(\varphi, \mathfrak{I})+\cdots \mathbb{V}_{i}(\varphi, \mathfrak{I}) .
\end{aligned}
$$


The approximate solution of the above example is

$$
\begin{aligned}
\mathbb{V}(\varphi, \mathfrak{I})= & e^{\varphi^{2}}+e^{\varphi^{2}}\{1+\wp \mathfrak{I}-\wp\}+e^{\varphi^{2}} \\
& \cdot\left\{(1-\wp) 2 \wp \mathfrak{I}+(1-\wp)^{2}+\frac{\wp^{2} \mathfrak{I}^{2}}{2}\right\} \\
& +e^{\varphi^{2}}\left\{(1-\wp)^{2} 3 \wp \mathfrak{I}+(1-\wp)^{3}+\frac{3 \wp^{2}(1-\wp) \mathfrak{J}^{2}}{2}+\frac{\wp^{3} \mathfrak{J}^{3}}{3 !}\right\}+\cdots
\end{aligned}
$$

Now, using the HPM, we get

$$
\sum_{i=0}^{\infty} p^{i} \mathbb{V}_{i}(\varphi, \mathfrak{\Im})=e^{\varphi^{2}}+p\left[\mathbb{Y}^{-1}\left\{(1+\wp(v-1)) \mathbb{Y}\left(\sum_{i=0}^{\infty} p^{i} H_{i}(w)\right)\right\}\right]
$$

Comparing the coefficients of power $p$, we have

$$
\begin{aligned}
p^{0}: \mathbb{V}_{0}(\varphi, \mathfrak{I}) & =e^{\varphi^{2}}, \\
p^{1}: \mathbb{V}_{1}(\varphi, \mathfrak{I}) & =\left[\mathbb{Y}^{-1}\left\{(1+\wp(v-1)) \mathbb{Y}\left(H_{0}(w)\right)\right\}\right]=e^{\varphi^{2}}\{1+\wp \mathfrak{I}-\wp\}, \\
p^{2}: \mathbb{V}_{2}(\varphi, \mathfrak{I}) & =\left[\mathbb{Y}^{-1}\left\{(1+\wp(v-1)) \mathbb{Y}\left(H_{1}(w)\right)\right\}\right] \\
& =e^{\varphi^{2}}\left\{(1-\wp) 2 \wp \mathfrak{I}+(1-\wp)^{2}+\frac{\wp^{2} \mathfrak{I}^{2}}{2}\right\}, \\
p^{3}: \mathbb{V}_{3}(\varphi, \mathfrak{I}) & =\left[\mathbb{Y}^{-1}\left\{(1+\wp(v-1)) \mathbb{Y}\left(H_{2}(w)\right)\right\}\right] \\
& =e^{\varphi^{2}}\left\{(1-\wp)^{2} 3 \wp \mathfrak{I}+(1-\wp)^{3}+\frac{3 \wp^{2}(1-\wp) \mathfrak{J}^{2}}{2}+\frac{\wp^{3} \mathfrak{J}^{3}}{3 !}\right\}
\end{aligned}
$$

Similarly, the remainder of the $\mathbb{V}_{i}(\varphi, \mathfrak{I})$ components for $n \geq 4$ can be completely achieved, thereby fully evaluating the series solutions. Finally, we estimate the approximate result $\mathbb{V}(\varphi, \mathfrak{I})$ by truncated sequence

$$
\begin{aligned}
\mathbb{V}(\varphi, \mathfrak{I})=\lim _{N \longrightarrow \infty} \sum_{n=1}^{N} \mathbb{V}_{i}(\varphi, \mathfrak{I}) \\
\mathbb{V}(\varphi, \mathfrak{I})=e^{\varphi^{2}}+e^{\varphi^{2}}\{1+\wp \mathfrak{I}-\wp\} \\
+e^{\varphi^{2}}\left\{(1-\wp) 2 \wp \mathfrak{\Im}+(1-\wp)^{2}+\frac{\wp^{2} \mathfrak{J}^{2}}{2}\right\} \\
+e^{\varphi^{2}}\left\{(1-\wp)^{2} 3 \wp \mathfrak{I}+(1-\wp)^{3}\right. \\
+ \\
\left.+\frac{3 \wp^{2}(1-\wp) \mathfrak{J}^{2}}{2}+\frac{\wp^{3} \mathfrak{J}^{3}}{3 !}\right\}+\cdots
\end{aligned}
$$

Figure 10 shows the analytical solution of two methods at different fractional-order $\wp=1,0.8,0.6$, and 0.4 of Example 13 . The special case for $\wp=1$, and the above problem close form is given as

$$
\mathbb{V}(\varphi, \mathfrak{J})=e^{\varphi^{2}+\mathfrak{J}^{2}}
$$

\section{Conclusion}

The homotopy perturbation transform technique and the Iterative transform method are used in this article to obtain numerical solutions for the fractional-order Cauchy-reaction diffusion equation, which is broadly used in applied sciences as a problem for spatial effects. In physical models, the techniques produce a series of form results that converge quickly. The obtained results in this article are expected to be useful for further analysis of complicated nonlinear physical problems. The calculations for these techniques are very simple and straightforward. As a result, we can conclude that these techniques can be used to solve a variety of nonlinear fractional-order partial differential equation schemes.

\section{Data Availability}

The numerical data used to support the findings of this study are included in the article.

\section{Conflicts of Interest}

The authors declare that there are no conflicts of interest regarding the publication of this article.

\section{References}

[1] S. Momani, "An algorithm for solving the fractional convection-diffusion equation with nonlinear source term," Communications in Nonlinear Science and Numerical Simulation, vol. 12, no. 7, pp. 1283-1290, 2007.

[2] Y. Liu and X. Zhao, "August. He's Variational Iteration Method for Solving Convection Diffusion Equations," in International Conference on Intelligent Computing, pp. 246-251, Springer, Berlin, Heidelberg, 2010.

[3] S. Yuzbasi and N. Sahin, "Numerical solutions of singularly perturbed one-dimensional parabolic convection-diffusion problems by the Bessel collocation method," Applied Mathematics and Computation, vol. 220, pp. 305-315, 2013.

[4] M. Ghasemia and K. M. Tavassoli, Application of He's homotopy perturbation method to solve a diffusion-convection problem, pp. 171-186, 2010.

[5] J. A. T. M. J. Sabatier, O. P. Agrawal, and J. T. Machado, Advances in Fractional Calculus, vol. 4, no. 9, 2007, Springer, Dordrecht, 2007.

[6] A. Atangana and D. Baleanu, "Caputo-Fabrizio derivative applied to groundwater flow within confined aquifer," Journal of Engineering Mechanics, vol. 143, no. 5, article D4016005, 2017.

[7] M. K. Alaoui, R. Fayyaz, A. Khan, R. Shah, and M. S. Abdo, "Analytical investigation of Noyes-field model for timefractional Belousov-Zhabotinsky reaction," Complexity, vol. 2021, 2021. 
[8] C. Baishya, S. J. Achar, P. Veeresha, and D. G. Prakasha, "Dynamics of a fractional epidemiological model with disease infection in both the populations. Chaos: an interdisciplinary," Journal of Nonlinear Science, vol. 31, no. 4, article 043130, 2021.

[9] H. Khan, R. Shah, P. Kumam, and M. Arif, "Analytical solutions of fractional order heat and wave equations by the natural transform decomposition method," Entropy, vol. 21, no. 6, p. 597, 2019.

[10] R. Shah, H. Khan, P. Kumam, M. Arif, and D. Baleanu, "Natural transform decomposition method for solving fractionalorder partial differential equations with proportional delay," Mathematics, vol. 7, no. 6, p. 532, 2019.

[11] H. Khan, R. Shah, D. Baleanu, P. Kumam, and M. Arif, “Analytical solution of fractional-order hyperbolic telegraph equation Using Natural Transform Decomposition Method," Electronics, vol. 8, no. 9, p. 1015, 2019.

[12] R. Shah, H. Khan, S. Mustafa, P. Kumam, and M. Arif, "Analytical solutions of fractional-order diffusion equations by natural transform decomposition method," Entropy, vol. 21, no. 6, p. $557,2019$.

[13] R. Shah, H. Khan, D. Baleanu, P. Kumam, and M. Arif, “A semi-analytical method to solve family of KuramotoSivashinsky equations," Journal of Taibah University for Science, vol. 14, no. 1, pp. 402-411, 2020.

[14] M. Madani, M. Fathizadeh, Y. Khan, and A. Yildirim, "On the coupling of the homotopy perturbation method and Laplace transformation," Mathematical and Computer Modelling, vol. 53, no. 9-10, pp. 1937-1945, 2011.

[15] Y. Khan and Q. Wu, "Homotopy perturbation transform method for nonlinear equations using He's polynomials," Computers \& Mathematics with Applications, vol. 61, no. 8, pp. 1963-1967, 2011.

[16] V. F. Morales-Delgado, J. F. Gomez-Aguilar, H. Ypez-Martnez, D. Baleanu, R. F. Escobar-Jimenez, and V. H. OlivaresPeregrino, "Laplace homotopy analysis method for solving linear partial differential equations using a fractional derivative with and without kernel singular," Advances in Difference Equations, vol. 2016, no. 1, 17 pages, 2016.

[17] Y. Li, B. T. Nohara, and S. Liao, "Series solutions of coupled Van der pol equation by means of homotopy analysis method," Journal of Mathematical Physics, vol. 51, no. 6, article 063517, 2010.

[18] Y. Keskin and G. Oturanc, "Reduced differential transform method for partial differential equations," International Journal of Nonlinear Sciences and Numerical Simulation, vol. 10, no. 6, pp. 741-750, 2009.

[19] P. K. Gupta, "Approximate analytical solutions of fractional Benney-Lin equation by reduced differential transform method and the homotopy perturbation method," Computers \& Mathematics with Applications, vol. 61, no. 9, pp. 2829-2842, 2011.

[20] N. H. Aljahdaly, R. P. Agarwal, R. Shah, and T. Botmart, "Analysis of the time fractional-order coupled burgers equations with non-singular kernel operators," Mathematics, vol. 9, no. 18, p. 2326, 2021.

[21] W. W. Mohammed, S. Albosaily, N. Iqbal, and M. El-Morshedy, "The effect of multiplicative noise on the exact solutions of the stochastic Burgers' equation," Waves in Random and Complex Media, vol. 1-13, 2021.

[22] A. U. K. Niazi, N. Iqbal, R. Shah, F. Wannalookkhee, and K. Nonlaopon, "Controllability for fuzzy fractional evolution equations in credibility space," Fractal and Fractional, vol. 5, no. 3, p. 112, 2021.

[23] P. Sunthrayuth, N. H. Aljahdaly, A. Ali, R. Shah, I. Mahariq, and A. M. Tchalla, " $\Phi$-Haar Wavelet Operational Matrix Method for Fractional Relaxation-Oscillation Equations Containing $\Phi$-Caputo Fractional Derivative," Journal of function spaces, vol. 2021, 2021.

[24] R. Shah, H. Khan, and D. Baleanu, "Fractional WhithamBroer-Kaup equations within modified analytical approaches," Axioms, vol. 8, no. 4, p. 125, 2019.

[25] W. W. Mohammed, N. Iqbal, A. Ali, and M. El-Morshedy, "Exact solutions of the stochastic new coupled Konno-Oono equation," Results in Physics, vol. 21, article 103830, 2021.

[26] G. D. Smith, G. D. Smith, and G. D. S. Smith, Numerical Solution of Partial Differential Equations: Finite Difference Methods, Oxford university press, 1985.

[27] N. Iqbal, R. Wu, and W. W. Mohammed, "Pattern formation induced by fractional cross-diffusion in a 3 -species food chain model with harvesting," Mathematics and Computers in Simulation, vol. 188, pp. 102-119, 2021.

[28] K. A. Touchent, Z. Hammouch, and T. Mekkaoui, “A modified invariant subspace method for solving partial differential equations with non-singular kernel fractional derivatives," Applied Mathematics and Nonlinear Sciences, vol. 5, no. 2, pp. 35-48, 2020.

[29] M. M. Khader, K. M. Saad, Z. Hammouch, and D. Baleanu, "A spectral collocation method for solving fractional KdV and KdV-burgers equations with non-singular kernel derivatives," Applied Numerical Mathematics, vol. 161, pp. 137-146, 2021.

[30] S. Rashid, A. Khalid, S. Sultana, Z. Hammouch, R. Shah, and A. M. Alsharif, "A novel analytical view of time-fractional Korteweg-De Vries equations via a new integral transform," Symmetry, vol. 13, no. 7, p. 1254, 2021.

[31] V. Daftardar-Gejji and H. Jafari, "An iterative method for solving nonlinear functional equations," Journal of Mathematical Analysis and Applications, vol. 316, no. 2, pp. 753763, 2006.

[32] H. Jafari, Iterative Methods for Solving System of Fractional Differential Equations, Pune University, 2006, [Ph.D. thesis].

[33] H. Jafari, M. Nazari, D. Baleanu, and C. M. Khalique, "A new approach for solving a system of fractional partial differential equations," Computers \& Mathematics with Applications, vol. 66, no. 5, pp. 838-843, 2013.

[34] M. A. Ramadan and M. S. Al-luhaibi, "New iterative method for solving the fornberg-whitham equation and comparison with homotopy perturbation transform method," Journal of Advances in Mathematics and Computer Science, vol. 4, no. 9, pp. 1213-1227, 2014.

[35] M. Naeem, A. M. Zidan, K. Nonlaopon, M. I. Syam, Z. AlZhour, and R. Shah, "A new analysis of fractional-order equal-width equations via novel techniques," Symmetry, vol. 13, no. 5, p. 886, 2021.

[36] L. Yan, "Numerical solutions of fractional Fokker-Planck equations using iterative Laplace transform method," Abstract and applied analysis, vol. 2013, Article ID 465160, 2013.

[37] J. H. He, "Homotopy perturbation technique," Computer Methods in Applied Mechanics and Engineering, vol. 178, no. 3-4, pp. 257-262, 1999.

[38] J. H. He, "Application of homotopy perturbation method to nonlinear wave equations," Chaos, Solitons \& Fractals, vol. 26, no. 3, pp. 695-700, 2005. 
[39] S. Das and P. K. Gupta, "An approximate analytical solution of the fractional diffusion equation with absorbent term and external force by homotopy perturbation method," Zeitschrift Fur Naturforschung A, vol. 65, no. 3, pp. 182-190, 2010.

[40] A. H. M. E. T. Yildirim, "An algorithm for solving the fractional nonlinear Schrodinger equation by means of the homotopy perturbation method," International Journal of Nonlinear Sciences and Numerical Simulation, vol. 10, no. 4, pp. 445-450, 2009.

[41] X. J. Yang, “A new integral transform method for solving steady heat-transfer problem," Thermal Science, vol. 20, suppl. 3, pp. 639-642, 2016.

[42] M. Caputo and M. Fabrizio, "On the singular kernels for fractional derivatives. Some applications to partial differential equations," Progress in Fractional Differentiation and Applications, vol. 7, no. 2, pp. 1-4, 2021.

[43] S. Ahmad, A. Ullah, A. Akgul, and M. De la Sen, "A novel Homotopy perturbation method with applications to nonlinear fractional order $\mathrm{KdV}$ and burger equation with exponential-decay kernel," Journal of Function Spaces, vol. 2021, 2021.

[44] Y. M. Chu, N. A. Shah, H. Ahmad, J. D. Chung, and S. M. Khaled, "A comparative study of semi-analytical methods for solving fractional-order Cauchy reaction-diffusion equation," Fractals, vol. 29, no. 6, pp. 2150143-2154148, 2021. 